\title{
Radiocarbon
}

\author{
1980
}

\section{GEOSECS ATLANTIC RADIOCARBON}

\author{
MINZE STUIVER \\ Quaternary Isotope Laboratory, University of Washington \\ Seattle, Washington 98195 \\ and \\ H G ÖSTLUND \\ Tritium Laboratory \\ Rosenstiel School of Marine and Atmospheric Sciences \\ University of Miami, Miami, Florida 33149
}

The program called Geochemical Ocean Section Study (GEOSECS) was initiated in 1971 and designed to make an oceanic inventory of chemical constituents in the world oceans, partly as a baseline study for future chemical changes, and partly to investigate large-scale oceanic transport and mixing processes. Measured were hydrographic parameters (salinity, temperature, and oxygen vs depth), the carbonate system, the nutrients, and assorted other constituents with heavy emphasis laid on the radioactive substances, radiocarbon, tritium, and radon.

The Atlantic expedition covered in this paper was undertaken in 1972-73, and a brief description of it was presented by Harmon Craig (1974). A companion paper by Östlund and Stuiver (this issue) lists the samples from the Pacific cruise.

Samples for ${ }^{14} \mathrm{C}$ were collected at 39 stations, usually around 18 samples per station, distributed through the entire water column. The station locations are seen on the track map, figure 1. On board the ship, the samples for radiocarbon determination were water drawn from the so-called 250-liter Gerard barrels and $\mathrm{CO}_{2}$ extracted by means of a modification of the method used by Fonselius and östlund (1959). In order to avoid carrying a large number of cylinders of compressed gas on board the ship, a circulating system was used with system pressure slightly above ambient at every point, with air recirculating between the purging of the acidified seawater sample and the absorption of $\mathrm{CO}_{2}$ in sodium hydroxide solution. The samples, usually around 0.5 moles of $\mathrm{CO}_{2}$, absorbed in excess $\mathrm{NaOH}$, were kept in well-sealed bottles and sent to laboratories for further processing. A short description of the sampling and measurement procedures, which also reports a cross-check between the laboratories, is available in a paper by Stuiver et al (1974).

Some Atlantic radiocarbon data are now available from earlier years: Fonselius and Östlund (1959) have presented a few measurements from the northernmost Atlantic in 1957, and Broecker and Olson (1961) released a rather comprehensive inventory as of 1955 through 1957. Of the 


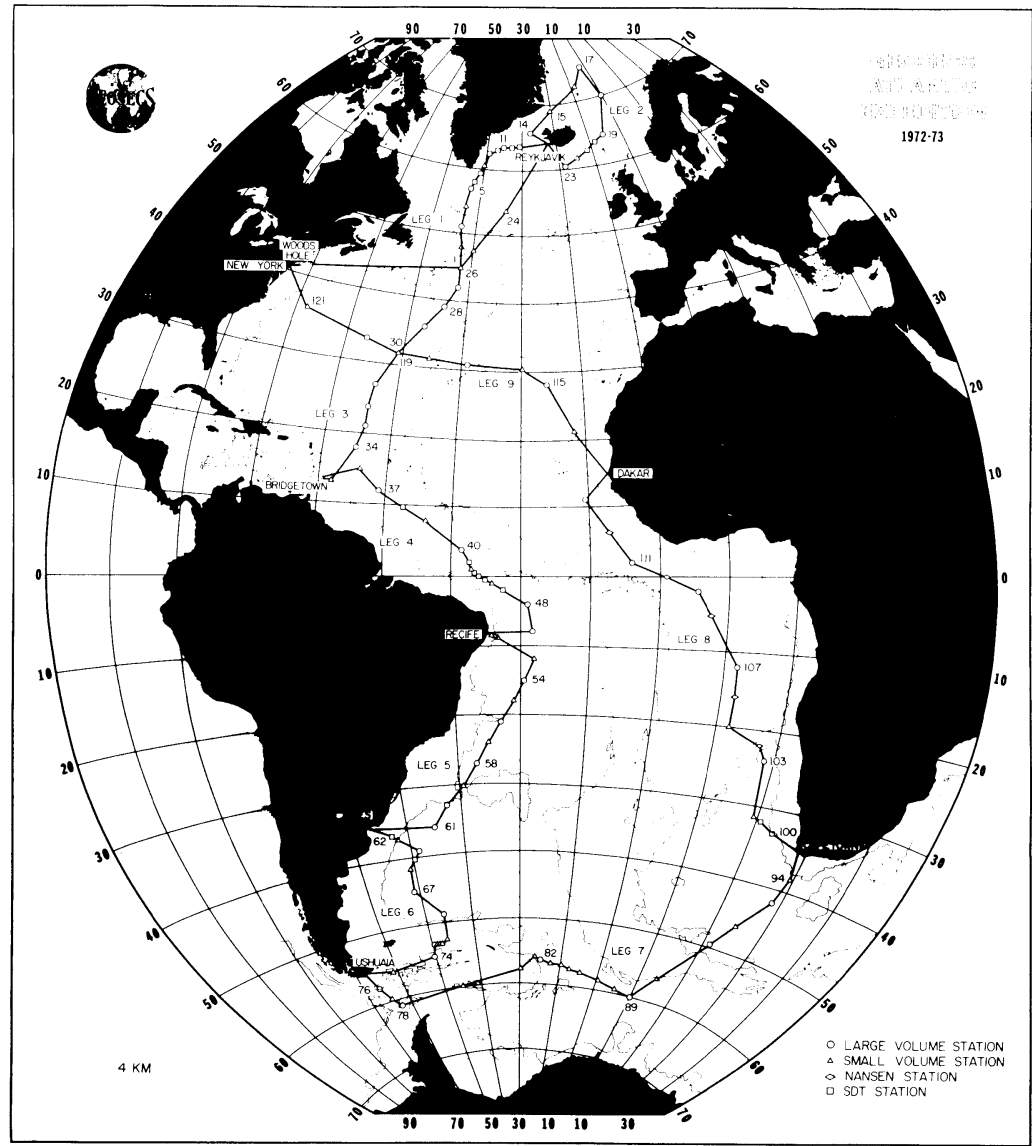

Fig 1. GEOSECS Atlantic Track 1972-1973.

data listed below, some have been released earlier by Östlund, Dorsey, and Rooth (1974), and by Stuiver and Robinson (1974). Average ${ }^{14} \mathrm{C}$ levels of Atlantic polar $\left(>50^{\circ} \mathrm{S}\right)$ gyre $\left(50^{\circ} \mathrm{S}-10^{\circ} \mathrm{S}\right.$ and $\left.10^{\circ} \mathrm{N}-50^{\circ} \mathrm{N}\right)$, and tropical waters $\left(10^{\circ} \mathrm{S}-10^{\circ} \mathrm{N}\right)$ are given in a SCOPE volume (Stuiver, Östlund, and McConnaughy, in press). Several papers of interpretative nature, primarily dealing with the process in the Atlantic deep waters, and with nuclear bomb ${ }^{14} \mathrm{C}$ additions, have been published or are in press. Among them: Stuiver (1976; in press), Broecker (1979), Broecker, Peng, and Stuiver (1978), Broecker and Takahashi (in press), Broecker, Peng, and Engh (in press), and Quay and Stuiver (in press).

THE $\Delta^{14}$ C SCALE

There has been some confusion concerning the $\Delta^{14} \mathrm{C}$ scale. In this paper, we have, in principle, followed the recommendations by Stuiver and Polach (1977), which means that the $\Delta^{14} \mathrm{C}$ values are recalculated to a standardized state of isotopic fractionation equivalent to standard 
wood $\left(\delta^{13} \mathrm{C}=-25 \%\right)$, and they relate to activities at time of sampling 1972. Appropriate corrections for the decay of the NBS ${ }^{14} \mathrm{C}$ standard have also been made. This scale is, for all practical purposes, the same as the one proposed by Broecker and Olson (1961).

EXPLANATION OF THE TABLES

All data on position, depths, and hydrography and total $\mathrm{CO}_{2}$ have been furnished by the GEOSECS Operations Group (now Physical and Chemical Ocean Data Facility) at Scripps Institution of Oceanography, which handled the logistics and serves as a temporary repository for all GEOSECS data. The following explains the column headings:

Position: Given in degrees and minutes. The ship frequently drifted during station time, so the position is defined to no better than \pm a few minutes.

SMPL \#: This is the operational sample number, in which the two last digits indicate the Gerard barrel number and the preceding digits the cast number. The first on station 27 is sample no. 1035: ic, cast \#10, Gerard \#35.

DEPTH M: Given in meters as calculated from density and pressure.

POT T DEG C: Potential temperature in degrees centigrade.

SAL $\%$ : Salinity in unit $\mathrm{g} / \mathrm{kg}$ seawater.

SIGMA THETA: Deviation from unity, in per mille, of the relative density in $\mathrm{g} / \mathrm{ml}$ where $\mathrm{ml}$ has the old value of $1.000027 \mathrm{~cm}^{3}$.

TCO2 $\mu \mathrm{M}$ : The total amount of inorganic carbon in $\mu$-moles per $\mathrm{kg}$ of seawater. All TCO2 data listed are still preliminary. At time of writing, a final re-evaluation is being made on all GEOSECS oceanic CO. measurements. This will most likely result in minor adjustments on these numbers.

DC14 $\%$ : This is $\Delta^{14} \mathrm{C}$ on the scale that was defined above. The accuracy is typically $\pm 4 \%$ and precision $\pm 3.5 \%$.

C14 LAB \#: This column lists ML for the Niami Laboratory and QL for the Washington Laboratory with numbers referring to our laboratory journals.

\section{THE SECTION}

In plate 1 , a vertical section of $\Delta^{14} \mathrm{C}$ in the Western Atlantic only is presented. The number of stations on the eastern track does not warrant a similar section. In addition, Stuiver (1978) has presented a more detailed section of the waters above 1000 meters. The horizontal scale in plate 1 is proportional to the distance between the stations along the track.

\section{ACKNOWLEDGMENTS}

This work was supported by National Science Foundation grants of the International Decade of Ocean Exploration division, grants GX28166, IDO 71-04200, OCE 76-03930 to the University of Washington, and grants GX-28165, IDO 71-04199, OCE 76-03050 to the University of Miami. Sample collection at sea was carried out by personnel of the GEOSECS Operations Group, with the late Arnold Bainbridge of the Scripps Institution of Oceanography directing the fieldwork. 


\section{REFFRENCES}

Broecker, W S, 1979, A revised estimate for the radiocarbon age of North Atlantic deep water: Jour Geophys Research, v 84, no. C6, p 3218-3226.

Brocker, W S and Olson, I A, 1961, Lamont radiocarbon measurements VIII: Radiocarbon, v 3, p 176-204.

Broecker, W S, Peng, T H, and Engh, Richard, 1980, Modeling the carbon system, in Stuiver, Minze and Kra, Rence, eds, International radiocarbon conference, 10th, Proc: Radiocarbon, v 22, no. 3.

Broecker, W S, Peng, T H, and Stuiver, Minze, 1978, An estimate of the upwelling rate in the equatorial Atlantic based on the distribution of bomb radiocarbon: Jour Geophys Research, v 83, p 6179-6186.

Broecker, W S and Takahashi, T, in press, Strategy for the use of bomb produced radiocarbon as a tracer for the uptake of fossil fuel CO. by the ocean's deep water source regions: Earth Planetary Sci Letters, in press.

Craig, Harmon, 1974, The GEOSECS program: 1972-1973: Earth Planetary Sci Letters, v 23, p 63-64.

Fonselius, S and Östlund, H G, 1959, Natural radiocarbon measurements on surface water from the North Atlantic and the Arctic Sea: Tellus, v 11, p 77-82.

Östlund, H G, Dorsey, H G, and Rooth, C G, 1974, GEOSECS North Atlantic radiocarbon and tritium results: Earth Planetary Sci Letters, v 23, p 69-86.

Östlund, H G and Stuiver, Minze, 1980, GEOSECS Pacific radiocarbon: Radiocarbon, v 22, no. 1 , p 25-53.

Quay, P D and Stuiver, Minze, 1980, Vertical advection-diffusion rates in the oceanic thermocline determined from bomb ${ }^{14} \mathrm{C}$ distribution, in Stuiver, Minze and Kra, Renee, eds, International radiocarbon conference, 10th, Proc: Radiocarbon, v 22, no. 2.

Stuiver, Minze, 1976, The ${ }^{14} \mathrm{C}$ distribution in west Atlantic abyssal waters: Earth Planetary Sci Letters, v 32, p 322-330.

Stuiver, Minze, 1978, Atmospheric carbon dioxide and carbon reservoir changes: Science, v 199, p 253-363.

Stuiver, Minze, in press, ${ }^{14} \mathrm{C}$ distribution in the Atlantic Ocean: Jour Geophys Research, in press.

Stuiver, Minze, Östlund, H G, and McConnaughy, T A, in press, GEOSECS Atlantic and Pacific ${ }^{14} \mathrm{C}$ distribution, in SCOPE Proc, La Jolla modeling conference, March 1979: New York, John Wiley \& Sons, in press.

Stuiver, Minze and Polach, H A, 1977, I)iscussion: Reporting of ${ }^{14} \mathrm{C}$ data: Radiocarbon, v 19 , p 253-258.

Stuiver, Minze and Robinson, S W, 1974, University of Washington GEOSECS North Atlantic Carbon-14 results: Earth Planctary Sci Letters, v 23, p 87-90.

Stuiver, Minze, Robinson, S IV Östlund, H G, and Dorsey, H G, 1974, Carbon-14 calibration between the University of Washington and the University of Miami GEOSECS laboratories: Earth Planctary Sci Letters, v 23, p 65-68. 


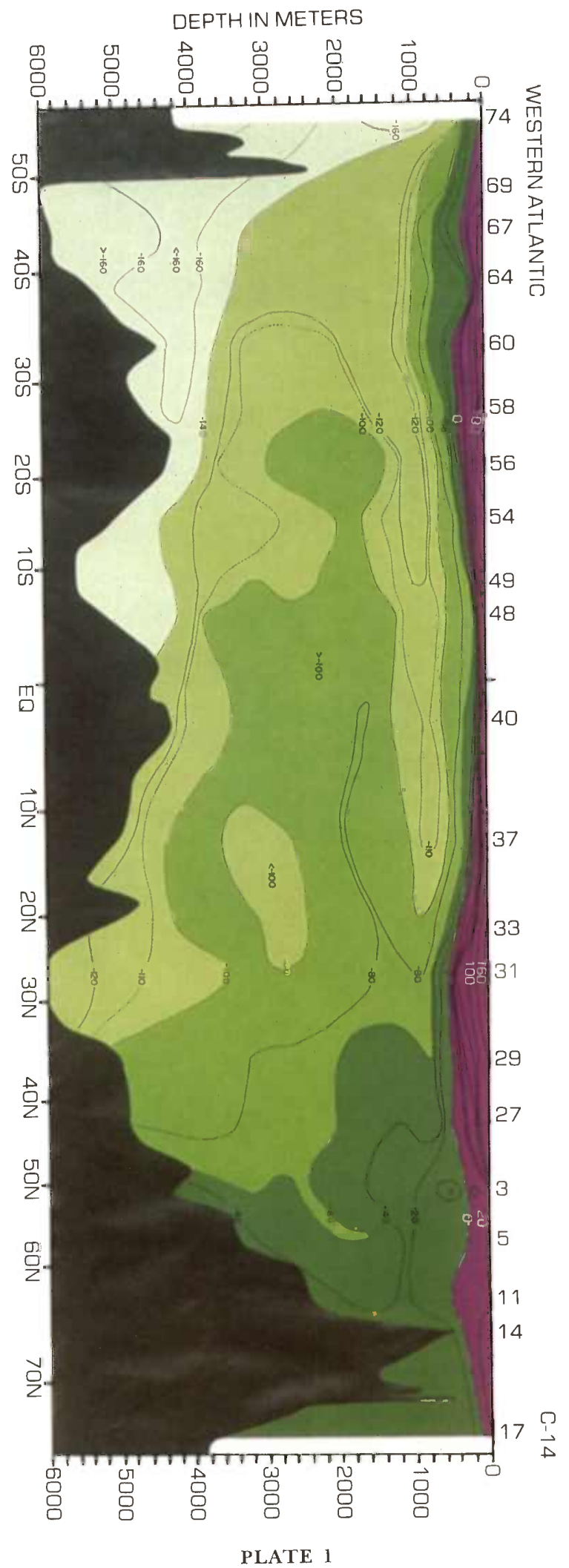

PLATE 1 


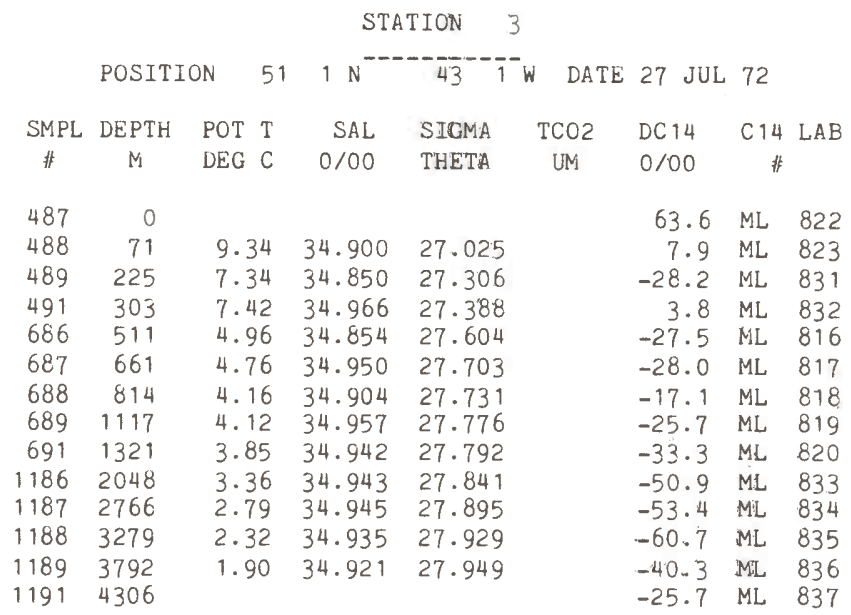

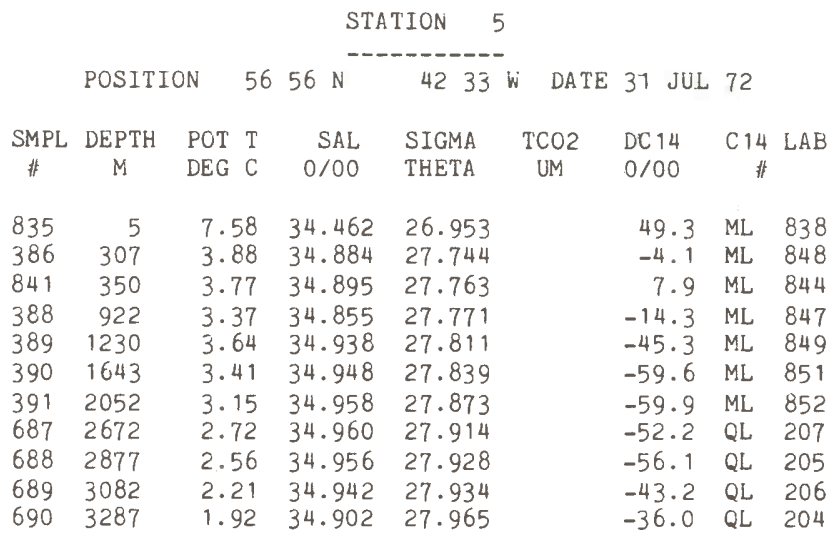




\begin{tabular}{|c|c|c|c|c|c|c|c|c|}
\hline & & & & IION & & & & \\
\hline & POSITI & 63 & $31 \mathrm{~N}$ & 3513 & DATE & $5 \mathrm{AUG}$ & 72 & \\
\hline SMPL & DEPTH & POT $\mathrm{T}$ & SAL & SIGMA & $\mathrm{TCO} 2$ & DC 14 & C 14 & LAB \\
\hline 非 & M & DEG C & $0 / 00$ & THETA & UM & $0 / 00$ & 非 & \\
\hline 86 & 8 & 8.86 & 34.955 & 27.146 & & 35.0 & ML & 853 \\
\hline 287 & 28 & 7.81 & 34.965 & 27.315 & & 29.9 & ML & 839 \\
\hline 288 & 100 & 6.50 & 35.040 & 27.5 & & 12.2 & $\mathrm{ML}$ & 840 \\
\hline 289 & 203 & 5.70 & 35.002 & 27.632 & & 10.1 & ML & 854 \\
\hline 290 & 305 & 5.06 & 34.962 & 27.677 & & 3.7 & ML & 855 \\
\hline & & & 4.944 & 27. & & & ML & 856 \\
\hline 588 & 612 & 4.19 & 34.920 & 27.741 & & $-4 \cdot 7$ & ML & 85 \\
\hline 587 & 819 & 3.91 & 34.909 & 27.761 & & -7.8 & ML & 858 \\
\hline 588 & 1026 & 3.76 & 34.913 & 27.778 & & -15.6 & ML & \\
\hline & 1232 & 3.69 & 34.922 & 27.794 & & -36.5 & ML & 860 \\
\hline 590 & 1440 & 3.67 & 34.945 & 27.813 & & -47.2 & ML & 861 \\
\hline & 1646 & 3.51 & 34.947 & 27.831 & & -50.1 & ML & \\
\hline & 1822 & 3.35 & 34.954 & 27.852 & & -48.9 & ML & \\
\hline & 2027 & 2.67 & 34.907 & 27.876 & & -39.0 & ML & \\
\hline $\begin{array}{l}888 \\
889\end{array}$ & $\begin{array}{l}2130 \\
2233\end{array}$ & $\begin{array}{l}2.27 \\
1.68\end{array}$ & $\begin{array}{l}34.909 \\
34.889\end{array}$ & $\begin{array}{l}27.913 \\
27.949\end{array}$ & & $\begin{array}{l}-35.9 \\
-27.2\end{array}$ & $\begin{array}{l}\text { ML } \\
\text { ML }\end{array}$ & $\begin{array}{l}006 \\
865\end{array}$ \\
\hline
\end{tabular}

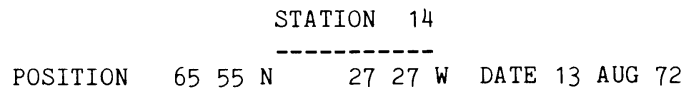

STATION 14

POSITION $6555 \mathrm{~N} 2727 \mathrm{~W}$ DATE 13 AUG 72

$\begin{array}{crrrrrrrr}\begin{array}{c}\text { SMPL } \\ \text { \# }\end{array} & \begin{array}{c}\text { DEPTH } \\ \text { M }\end{array} & \begin{array}{r}\text { POT T } \\ \text { DEG C }\end{array} & \begin{array}{r}\text { SAL } \\ 0 / 00\end{array} & \begin{array}{c}\text { SIGMA } \\ \text { THETA }\end{array} & \begin{array}{c}\text { TC02 } \\ \text { UM }\end{array} & \begin{array}{r}\text { DC } 14 \\ 0 / 00\end{array} & \begin{array}{c}\text { C14 } \\ \text { \# }\end{array} \\ 386 & 7 & 1.04 & 30.121 & 24.159 & & 67.6 & \text { ML } & 1197 \\ 387 & 83 & -0.83 & 33.122 & 26.654 & & 45.3 & \text { ML } & 1192 \\ 388 & 214 & 2.19 & 34.531 & 27.614 & & 19.4 & \text { ML } 1196 \\ 389 & 316 & -0.38 & 34.605 & 27.834 & & 16.7 & \text { ML } & 1195 \\ 390 & 417 & 0.66 & 34.784 & 27.923 & & -4.1 & \text { ML } 1194 \\ 391 & 604 & -0.29 & 34.925 & 28.088 & & -28.4 & \text { ML } 1193\end{array}$




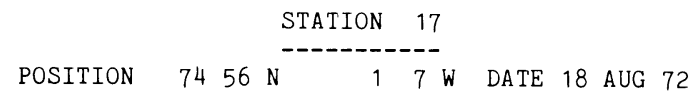

\begin{tabular}{|c|c|c|c|c|c|c|c|c|}
\hline SMPL & DEPTH & POT T & SAL & SIGMA & $\mathrm{TCO} 2$ & DC 14 & C 14 & $\mathrm{LAB}$ \\
\hline \# & M & DEG C & $0 / 00$ & THETA & UM & $0 / 00$ & 非 & \\
\hline 386 & 4 & 4.57 & 34.500 & 27.366 & & 33.9 & ML & 872 \\
\hline 387 & 40 & -0.91 & 34.774 & 27.993 & & 8.8 & ML & 881 \\
\hline $\begin{array}{l}388 \\
390\end{array}$ & $\begin{array}{r}76 \\
230\end{array}$ & $\begin{array}{l}-1.68 \\
-1.42\end{array}$ & $\begin{array}{l}34.826 \\
34.861\end{array}$ & $\begin{array}{l}28.061 \\
28.081\end{array}$ & & $\begin{array}{r}10.2 \\
-11.6\end{array}$ & $\begin{array}{l}\text { ML } \\
\text { ML }\end{array}$ & $\begin{array}{l}879 \\
880\end{array}$ \\
\hline 391 & 306 & -1.22 & 34.870 & 28.081 & & $-29 \cdot 3$ & ML & 873 \\
\hline 1086 & 511 & -1.08 & 34.892 & 28.095 & & -22.7 & ML & 883 \\
\hline 1087 & 820 & -1.09 & 34.892 & 28.095 & & -49.2 & ML & 884 \\
\hline 1088 & 1025 & -1.13 & 34.902 & 28.104 & & -57.9 & ML & 885 \\
\hline 1089 & 1332 & -1.17 & 34.899 & 28.103 & & -48.1 & ML & 886 \\
\hline 1386 & 2079 & -1.27 & 34.892 & 28.101 & & -45.2 & ML & 887 \\
\hline 1387 & 2386 & -1.29 & 34.892 & 28.102 & & -54.4 & ML & 888 \\
\hline 588 & 2538 & $-1 \cdot 30$ & 34.892 & 28.102 & & -55.3 & ML & 867 \\
\hline 1388 & 2592 & $-1 \cdot 30$ & 34.894 & 28.103 & & -50.4 & ML & 890 \\
\hline 1389 & 3055 & $-1 \cdot 31$ & 34.890 & 28.101 & & -55.5 & ML & 889 \\
\hline 589 & 3070 & $-1 \cdot 31$ & 34.890 & 28.101 & & -49.6 & ML & 875 \\
\hline 1390 & 3261 & $-1 \cdot 31$ & 34.893 & 28.100 & & -47.6 & ML & 091 \\
\hline 590 & 3352 & $-1 \cdot 31$ & 34.894 & 28.105 & & -52.9 & ML & 868 \\
\hline 1391 & 3416 & $-1 \cdot 31$ & 34.898 & 28.107 & & -50.4 & ML & 892 \\
\hline 591 & 3552 & $-1 \cdot 31$ & 34.893 & 28.104 & & -48.4 & ML & 1 \\
\hline
\end{tabular}

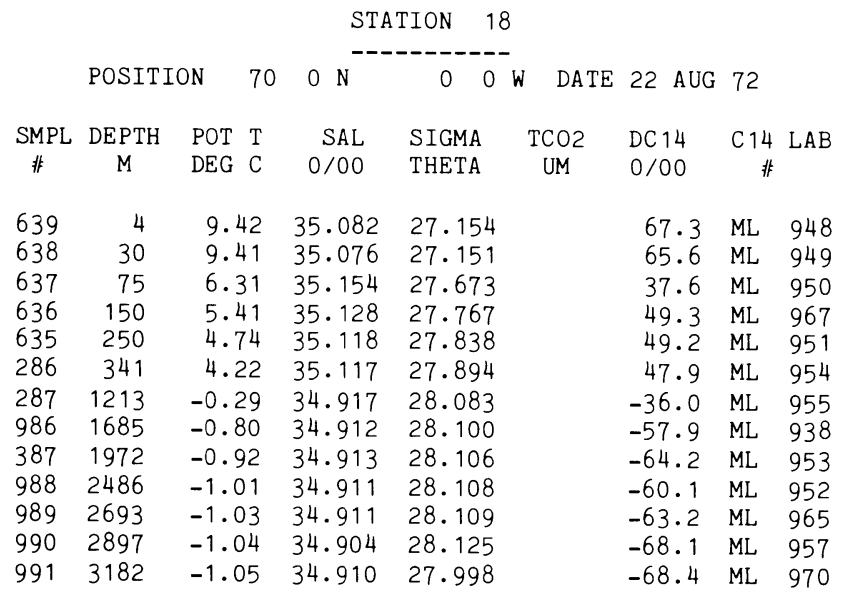




\begin{tabular}{|c|c|c|c|c|c|c|c|c|}
\hline & & & & CON & & & & \\
\hline & POSITI & $\mathrm{ON}$ & $12 \mathrm{~N}$ & 534 & DATE & $24 \mathrm{AUG}$ & 72 & \\
\hline$\underset{\not ⿰}{\text { SMPL }}$ & $\begin{array}{c}\text { DEPTH } \\
M\end{array}$ & $\begin{array}{ll}\text { POT } & T \\
\text { DEG } & \text { C }\end{array}$ & $\begin{array}{r}\text { SAL } \\
0 / 00\end{array}$ & $\begin{array}{l}\text { SIGMA } \\
\text { THETA }\end{array}$ & $\begin{array}{c}\text { TC02 } \\
\text { UM }\end{array}$ & $\begin{array}{l}\text { DC } 14 \\
0 / 00\end{array}$ & 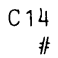 & LAB \\
\hline 286 & 4 & 10.41 & 35.053 & 26.962 & & 47.3 & ML & 894 \\
\hline 287 & 31 & $10 \cdot 30$ & 35.053 & 26.982 & & 54.7 & ML & 895 \\
\hline 288 & 66 & 8.24 & 35.116 & 27.360 & & 26.9 & ML & \\
\hline 289 & 144 & 5.13 & 35.013 & 27.709 & & 43.7 & ML & 897 \\
\hline 290 & 247 & 3.59 & 34.905 & 27.789 & & 41.3 & ML & 899 \\
\hline 291 & 349 & 2.01 & 34.928 & 27.947 & & 38.3 & ML & 898 \\
\hline 986 & 458 & 1.88 & 35.010 & 28.023 & & 28.4 & ML & 9 \\
\hline 987 & 558 & 1.51 & 34.998 & 28.041 & & 25.4 & ML & \\
\hline 988 & 663 & 0.98 & 34.968 & 28.052 & & 4.7 & ML & 908 \\
\hline 989 & 765 & 0.38 & 34.934 & 28.063 & & -12.8 & ML & 909 \\
\hline 990 & 866 & 0.06 & 34.926 & 28.072 & & -30.8 & ML & 910 \\
\hline 991 & 969 & -0.20 & 34.914 & 28.077 & & -37.4 & ML & 841 \\
\hline 486 & 1113 & -0.59 & 34.913 & 28.093 & & -54.6 & ML & 900 \\
\hline 487 & 1532 & -0.86 & 34.912 & 28.102 & & -62.9 & ML & \\
\hline 488 & 2046 & -0.99 & 34.913 & 28.108 & & $-68 \cdot 3$ & ML & 904 \\
\hline 489 & 2455 & -1.03 & 34.911 & 28.108 & & -67.0 & ML & \\
\hline 490 & 2866 & -1.04 & 34.911 & 28.109 & & -68.2 & ML & \\
\hline 491 & 3278 & -1.04 & 34.914 & 28.111 & & -68.4 & ML & \\
\hline
\end{tabular}

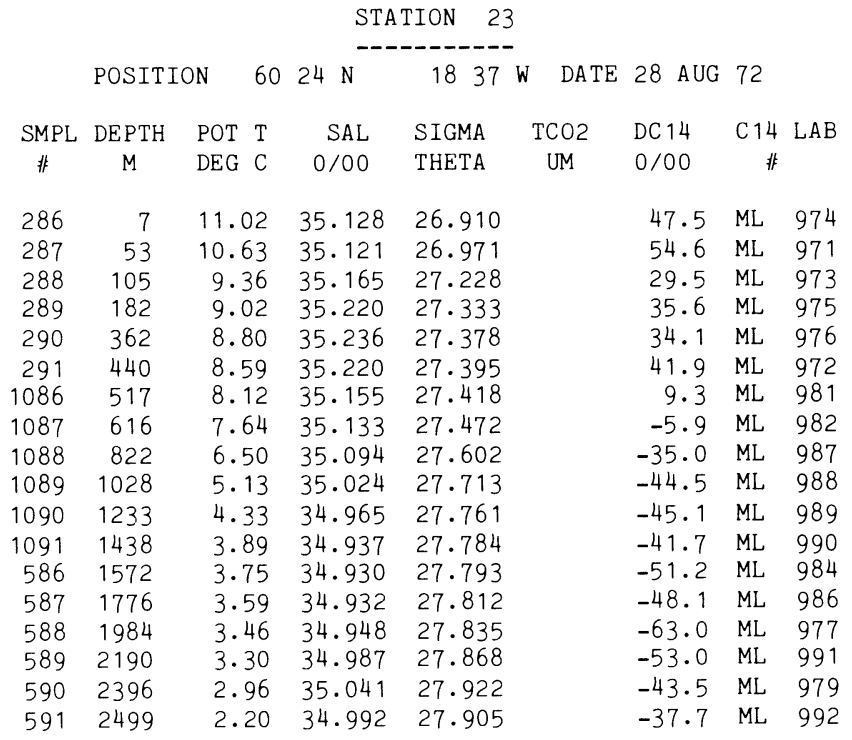




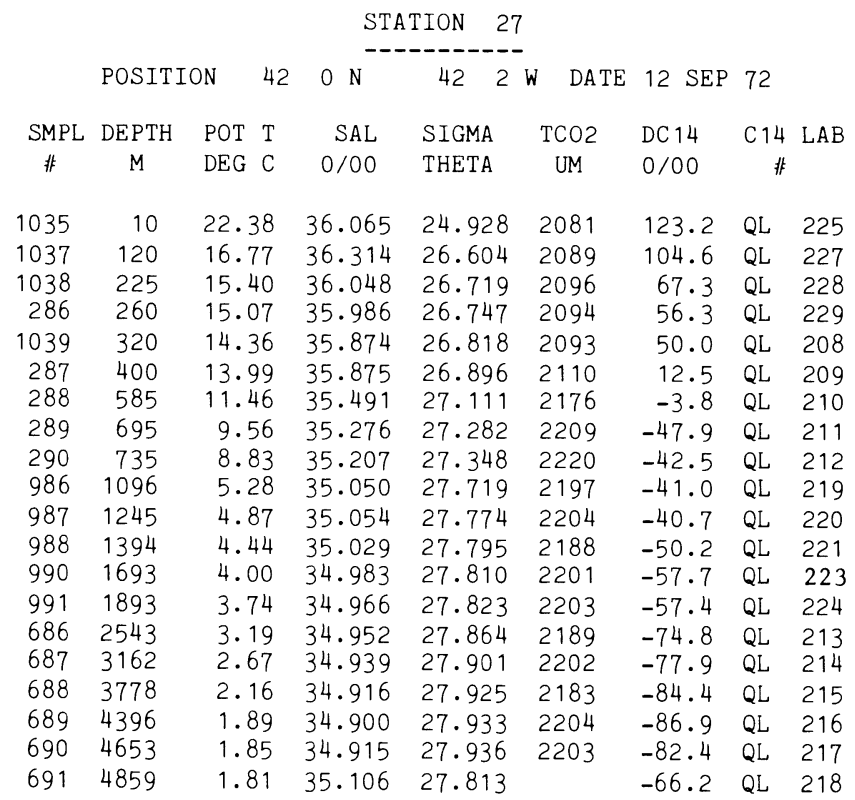

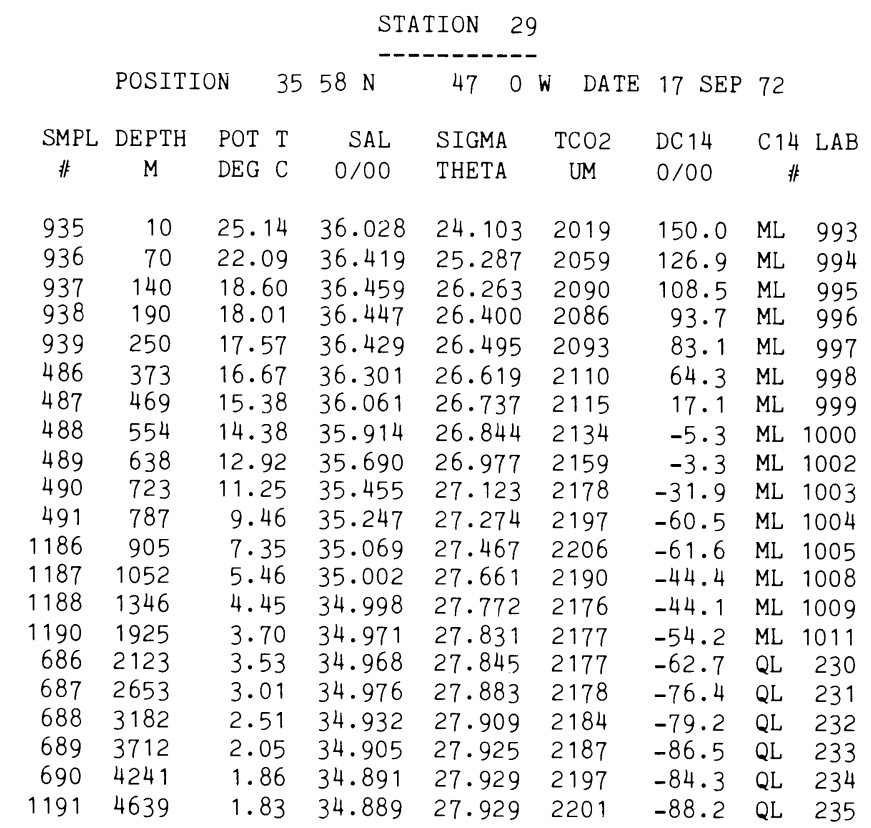


STATION 31

POSITION $27 \quad 0 \mathrm{~N} \quad 5332 \mathrm{~W}$ DATE 22 SEP 72

\begin{tabular}{|c|c|c|c|c|c|c|c|c|}
\hline SMPL & DEPTH & POT $\mathrm{T}$ & SAL & SIGMA & TCO2 & DC 14 & C 14 & LAB \\
\hline \# & M & DEG C & $0 / 00$ & THETA & UM & $0 / 00$ & 非 & \\
\hline 935 & 10 & 27.29 & 36.908 & 24.102 & 2046 & 185.7 & ML & 927 \\
\hline 936 & 70 & 22.27 & 36.825 & 25.550 & 2085 & 159.7 & ML & \\
\hline 937 & 100 & 1. & 6.943 & 25.939 & 2098 & 158.4 & ML & $y$ \\
\hline 939 & 240 & & 36.442 & +67 & 2103 & 110.2 & ML & \\
\hline 940 & 320 & 2 & 398 & 26.604 & 210 & .3 & ML & \\
\hline 1086 & 422 & 2 & 344 & 639 & 209 & & ML & \\
\hline 087 & 618 & 3. & 35.788 & & 2155 & -1 & ML & \\
\hline 1088 & 816 & 9.45 & 35.307 & 27.324 & 2205 & -65.2 & ML & 63 \\
\hline 1089 & 914 & & 35. & 27. & 221 & -8 & ML & 67 \\
\hline 1090 & 1013 & & & 27.5 & 221 & & ML & \\
\hline 1091 & 1261 & 5.50 & 35.127 & 27.757 & 2196 & -72.4 & ML & 964 \\
\hline 486 & 1486 & 4.70 & 35.098 & 27.826 & 2197 & -78.1 & ML & 920 \\
\hline 487 & 1735 & 0 & 35.065 & 27.865 & 2195 & -84.4 & ML & 21 \\
\hline 488 & 1985 & .56 & 35.020 & 27.883 & 2194 & -85.2 & ML & 22 \\
\hline 489 & 2234 & .18 & 34 & 27.896 & & & ML & \\
\hline 490 & 2484 & & & & & & ML & \\
\hline 491 & 2882 & 2.64 & 34.943 & $27 \cdot 907$ & 2207 & -99.0 & ML & 25 \\
\hline 286 & 3013 & 57 & 34.938 & 27.909 & 2206 & $-101 \cdot 5$ & ML & 311 \\
\hline 287 & 3512 & & & & & & ML & 12 \\
\hline 288 & 4011 & 1.90 & 34.884 & 27.919 & 2217 & -105.0 & ML & 914 \\
\hline 289 & 4509 & 1.72 & 34.869 & 27.921 & 2221 & -108.6 & ML & \\
\hline 290 & 5008 & & & 27.922 & 2226 & -114.0 & ML & 916 \\
\hline 291 & 5508 & 1.59 & 34.860 & 27.923 & 2226 & -120.8 & ML & 9 \\
\hline
\end{tabular}

\begin{tabular}{|c|c|c|c|c|c|c|c|c|}
\hline & & & STA & ION 33 & & & & \\
\hline & POSIT & ON & $0 \mathrm{~N}$ & $\begin{array}{ll}54 & 0\end{array}$ & DATE & 26 SEP & 72 & \\
\hline SMPL & DEPTH & POT T & SAL & SIGMA & TCO2 & DC 14 & C 14 & $\mathrm{LAB}$ \\
\hline 非 & M & DEG C & $0 / 00$ & THETA & UM & $0 / 00$ & 非 & \\
\hline 1135 & 15 & 27.17 & 36.084 & 23.508 & & 164.7 & ML & 983 \\
\hline 1136 & 70 & 24.82 & 37.295 & 25.159 & 2087 & 148.7 & $Q L$ & 306 \\
\hline 1137 & 100 & 23.02 & 37.306 & 25.699 & 2113 & 159.0 & QL & 307 \\
\hline 1138 & 160 & 20.92 & 37.060 & 26.101 & 2106 & 114.9 & QL & 308 \\
\hline 1139 & 240 & 17.94 & 36.551 & 26.497 & 2142 & 63.2 & QL & 309 \\
\hline 1140 & 320 & 16.36 & 36.278 & 26.675 & 2151 & 36.4 & $Q L$ & 310 \\
\hline 1086 & 396 & 15.00 & 36.043 & 26.805 & 2155 & 10.2 & $\mathrm{QL}$ & 300 \\
\hline 1087 & 451 & 14.02 & 35.895 & 26.907 & 2168 & -23.2 & QL & 301 \\
\hline 1088 & 531 & 12.63 & 35.703 & 27.047 & 2184 & -44.9 & $\mathrm{QL}$ & 302 \\
\hline 1089 & 606 & 11.28 & 35.525 & $27 \cdot 170$ & 2196 & -62.1 & QL & 303 \\
\hline 1090 & 688 & 9.63 & $35 \cdot 312$ & 27.299 & 2217 & -73.6 & $\mathrm{QL}$ & 304 \\
\hline $\begin{array}{r}1091 \\
486\end{array}$ & $\begin{array}{l}763 \\
892\end{array}$ & $\begin{array}{l}8.37 \\
6.75\end{array}$ & $\begin{array}{l}35.170 \\
35.012\end{array}$ & $\begin{array}{l}27.392 \\
27.502\end{array}$ & $\begin{array}{l}2222 \\
2237\end{array}$ & $\begin{array}{l}-85.7 \\
-92.2\end{array}$ & $\begin{array}{l}\text { QL } \\
Q L\end{array}$ & $\begin{array}{l}305 \\
297\end{array}$ \\
\hline 487 & 995 & 5.86 & 34.941 & 27.564 & 2237 & -98.2 & $\mathrm{QL}$ & 298 \\
\hline 488 & 1093 & 5.42 & 34.946 & 27.623 & 2225 & $-93 \cdot 3$ & QL & 299 \\
\hline 489 & 1493 & $4 \cdot 32$ & 35.014 & 27.801 & 2202 & -80.6 & $\mathrm{QL}$ & 267 \\
\hline 490 & 1842 & 3.76 & 35.015 & 27.860 & 2203 & -84.4 & $\mathrm{QL}$ & 268 \\
\hline 491 & 2238 & 3.15 & 34.980 & 27.890 & 2194 & $-94 \cdot 1$ & QL & 269 \\
\hline 286 & 2702 & 2.67 & 34.945 & 27.906 & 2207 & -105.9 & ML & 926 \\
\hline 287 & 3200 & 2.37 & 34.924 & 27.914 & 2207 & -96.3 & $\mathrm{QL}$ & 270 \\
\hline 288 & 3697 & 2.09 & 34.906 & 27.922 & 2221 & -99.0 & $Q L$ & 271 \\
\hline 289 & 4195 & 1.86 & 34.889 & 27.927 & 2219 & -97.7 & QL & 272 \\
\hline 290 & 4682 & 1.60 & 34.858 & 27.916 & 2238 & -113.6 & QL & 273 \\
\hline 291 & 5191 & 1.47 & 34.090 & 27.917 & & $-113 \cdot 7$ & QL & 274 \\
\hline
\end{tabular}




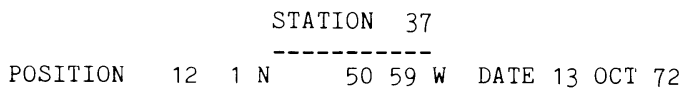

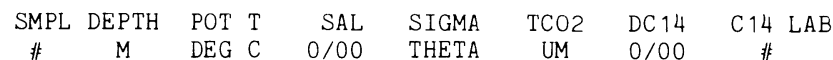

$\begin{array}{rrrrrrrrr}835 & 5 & 28.04 & 35.910 & 23.116 & & 132.3 & \text { ML } & 1012 \\ 836 & 75 & 24.45 & 36.571 & 24.723 & 2050 & 136.6 & \text { ML } & 1013 \\ 1086 & 101 & 22.39 & 36.704 & 25.420 & 2089 & 91.7 & \text { ML } & 1014 \\ 1087 & 162 & 16.21 & 36.162 & 26.604 & 2174 & 35.2 & \text { ML } & 1015 \\ 1088 & 306 & 10.14 & 35.171 & 27.091 & 2202 & -59.0 & \text { ML } & 1016 \\ 1089 & 408 & 9.04 & 34.993 & 27.146 & 2206 & -71.2 & \text { ML } & 1018 \\ 1090 & 510 & 7.69 & 34.802 & 27.205 & 2226 & -90.2 & \text { ML } & 1019 \\ 1091 & 712 & 6.16 & 34.669 & 27.308 & 2234 & -110.7 & \text { ML } & 1020 \\ 586 & 894 & 5.13 & 34.653 & 27.418 & 2239 & -110.3 & \text { ML } & 1021 \\ 587 & 993 & 5.02 & 34.717 & 27.489 & 2232 & -109.4 & \text { ML } & 1022 \\ 588 & 1094 & 5.04 & 34.837 & 27.580 & 2227 & -94.7 & \text { ML } & 1023 \\ 589 & 1392 & 4.49 & 34.977 & 27.753 & 2192 & -90.6 & \text { ML } & 1025 \\ 590 & 1690 & 3.70 & 34.986 & 27.843 & 2178 & -78.3 & \text { ML } & 1026 \\ 591 & 1983 & 3.30 & 34.965 & 27.866 & 2178 & -80.4 & \text { ML } & 1024 \\ 289 & 2441 & 2.85 & 34.947 & 27.891 & 2183 & -91.6 & \text { QL } & 275 \\ 290 & 2959 & 2.47 & 34.924 & 27.905 & 2198 & -101.6 & \text { QL } & 276 \\ 291 & 3472 & 2.20 & 34.908 & 27.915 & 2197 & -105.9 & \text { QL } & 277 \\ 292 & 3985 & 1.96 & 34.893 & 27.922 & 2196 & -90.2 & \text { QL } & 278 \\ 293 & 4499 & 1.79 & 34.880 & 27.923 & 2207 & -104.1 & \text { QL } & 279 \\ 294 & 5014 & 1.27 & 34.614 & 27.625 & 2236 & -125.3 & \text { QL } & 280\end{array}$

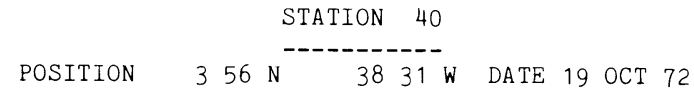

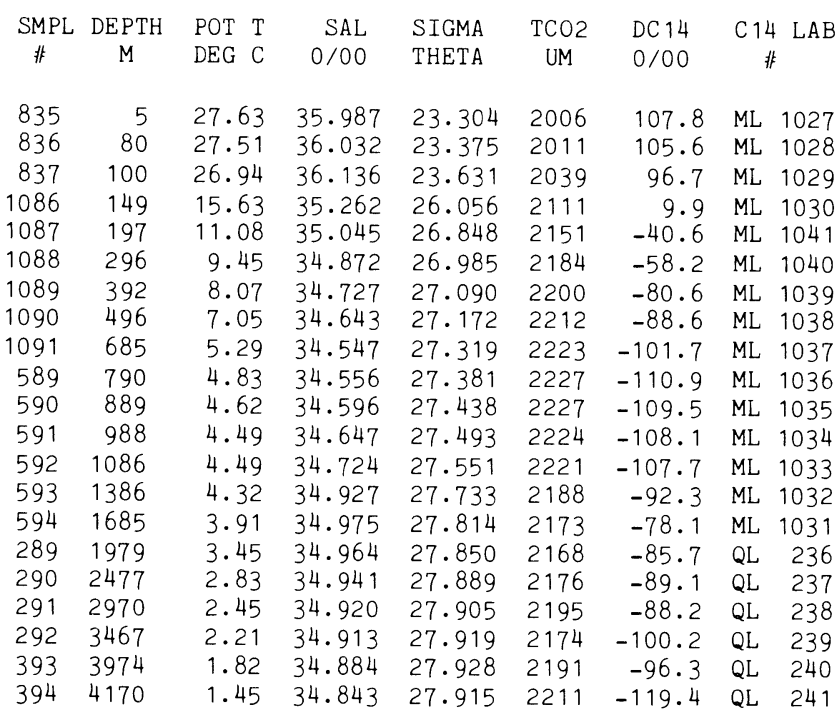




\begin{tabular}{|c|c|c|c|c|c|c|c|c|}
\hline & \multicolumn{8}{|c|}{ STATION 48} \\
\hline & \multicolumn{2}{|c|}{ POSITION } & $0 \mathrm{~S}$ & 29 & DATE & 25 OCT & 72 & \\
\hline SMPL & DEPTH & POT T & SAL & SIGMA & TCO2 & DC 14 & C 14 & LAB \\
\hline 非 & M & DEG C & $0 / 00$ & THETA & UM & $0 / 00$ & 非 & \\
\hline 635 & 5 & 26.22 & 36.080 & 23.814 & & 83.0 & QL & 349 \\
\hline 636 & 70 & 25.82 & 36.064 & 23.982 & 2038 & 72.5 & QL & 352 \\
\hline 637 & 100 & 21.09 & 36.447 & 25.589 & 2099 & 84.6 & QL & 353 \\
\hline 638 & 160 & 13.21 & 35.317 & 26.631 & 2156 & -34.9 & QL & 354 \\
\hline 1187 & 195 & 11.31 & 35.082 & 26.818 & 2187 & -63.2 & QL & 355 \\
\hline 1188 & 295 & 9.42 & 34.832 & 26.958 & 2173 & $-79 \cdot 3$ & QL & 356 \\
\hline & 395 & 7.65 & 34.669 & 27.107 & 22 & & QL & 35 \\
\hline 1190 & 495 & 6.28 & 34.550 & 27.201 & 2227 & -98.5 & QL & 358 \\
\hline 1191 & 673 & 4.71 & 34.461 & 27.320 & 2226 & -11 & QL & 359 \\
\hline 1193 & 840 & 4.23 & 34.499 & 27.402 & 2230 & $-113 \cdot 2$ & QL & 360 \\
\hline 486 & 1070 & 4.13 & 34.653 & 27.534 & 2216 & $-112 \cdot 3$ & QL & 361 \\
\hline 487 & 1217 & 4.27 & 34.816 & 27.646 & 2210 & $-100 \cdot 3$ & QL & 362 \\
\hline 488 & 1367 & 4.24 & 34.883 & 27.711 & 2202 & -93.4 & QL & 363 \\
\hline 490 & 1804 & 3.58 & 34.958 & 27.833 & 2177 & -88.7 & ML & 1064 \\
\hline 492 & 2259 & 3.05 & 34.939 & 27.867 & 2181 & .4 & ML & 1063 \\
\hline 494 & 2652 & 2.68 & 34.921 & 27.886 & 2192 & & ML & \\
\hline 287 & 3009 & 2.44 & 34.917 & 27.903 & 2189 & -96.5 & QL & 242 \\
\hline 288 & 3408 & 2.28 & 34.911 & $27 \cdot 912$ & 2191 & -99.1 & $\mathrm{QL}$ & 243 \\
\hline 289 & 3705 & 2.11 & 34.900 & 27.917 & 2192 & & QL & 24 \\
\hline 290 & 4101 & 1.22 & 34.807 & 27.907 & 2231 & -129.4 & QL & 245 \\
\hline 292 & 4497 & 0.59 & 34.741 & 27.893 & 2253 & -147.9 & QL & 246 \\
\hline 293 & 5031 & 0.21 & 34.699 & 27.881 & 2273 & -155.1 & QL & 247 \\
\hline
\end{tabular}

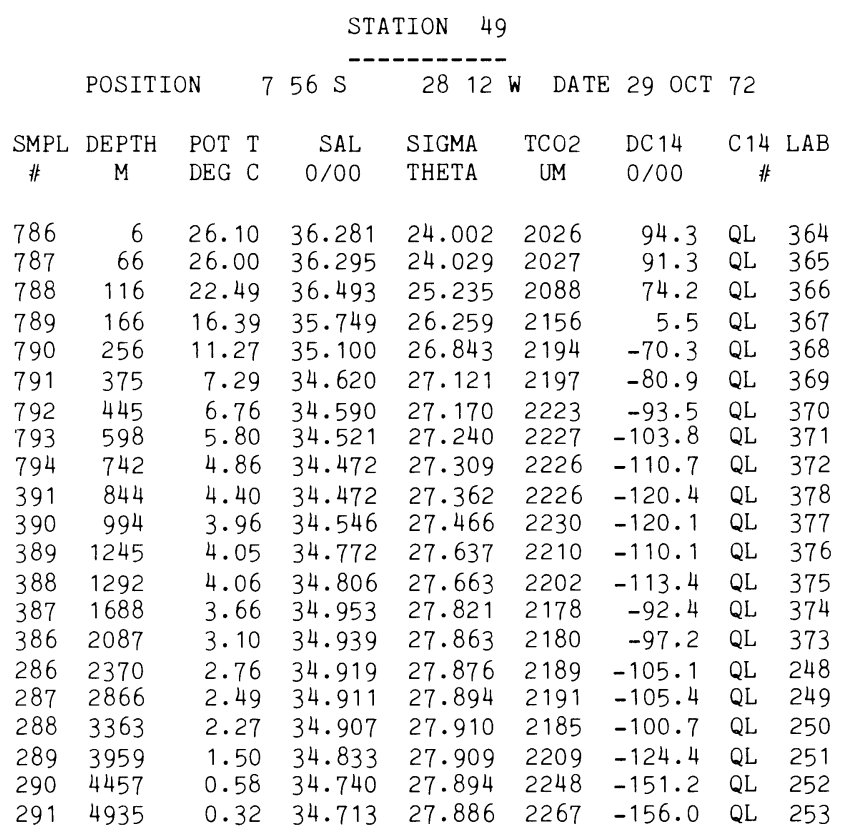




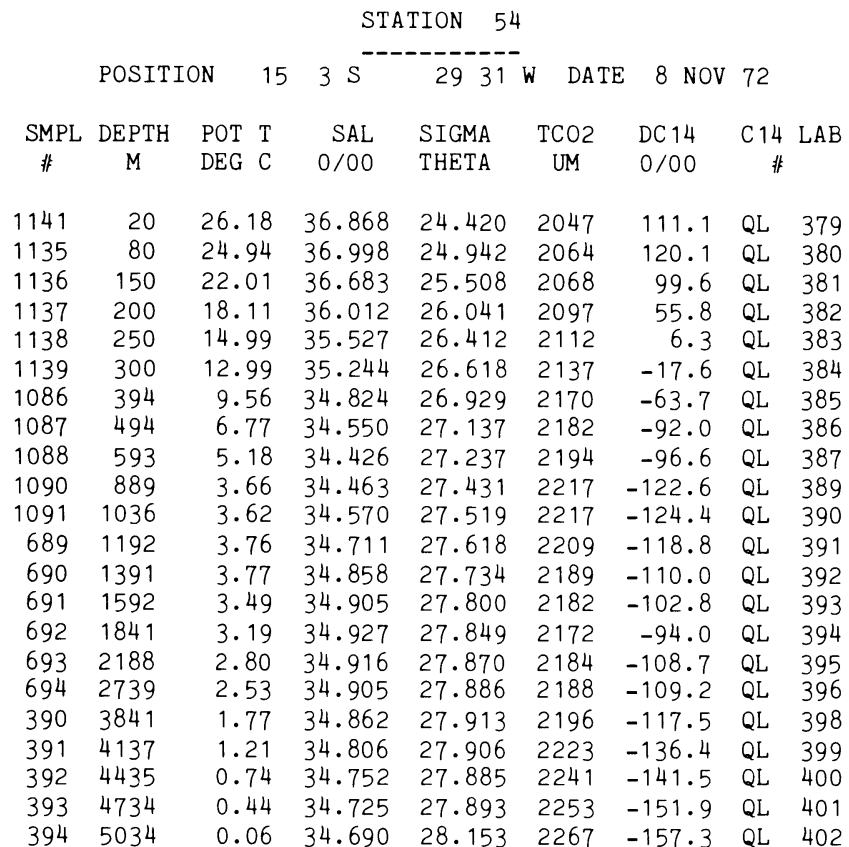

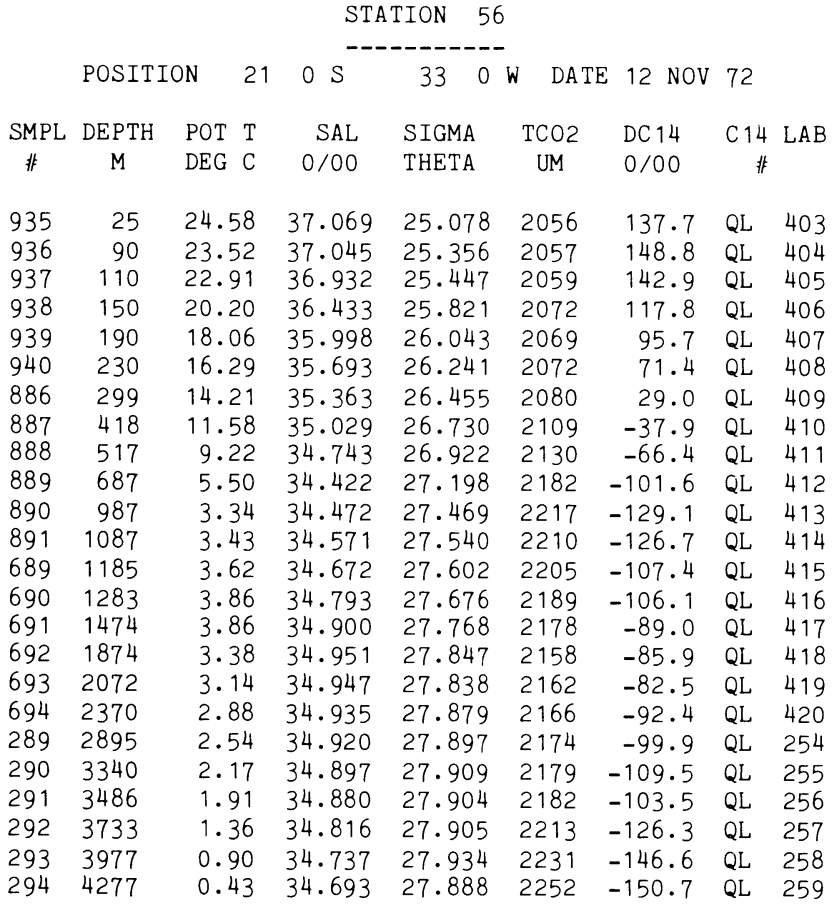




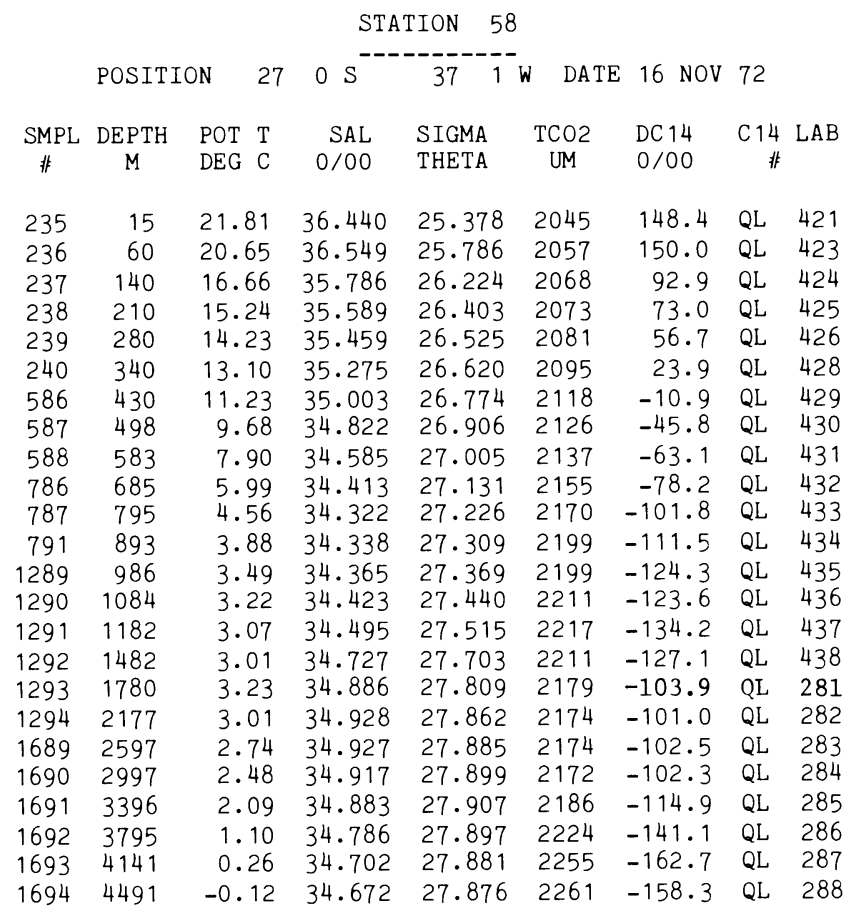

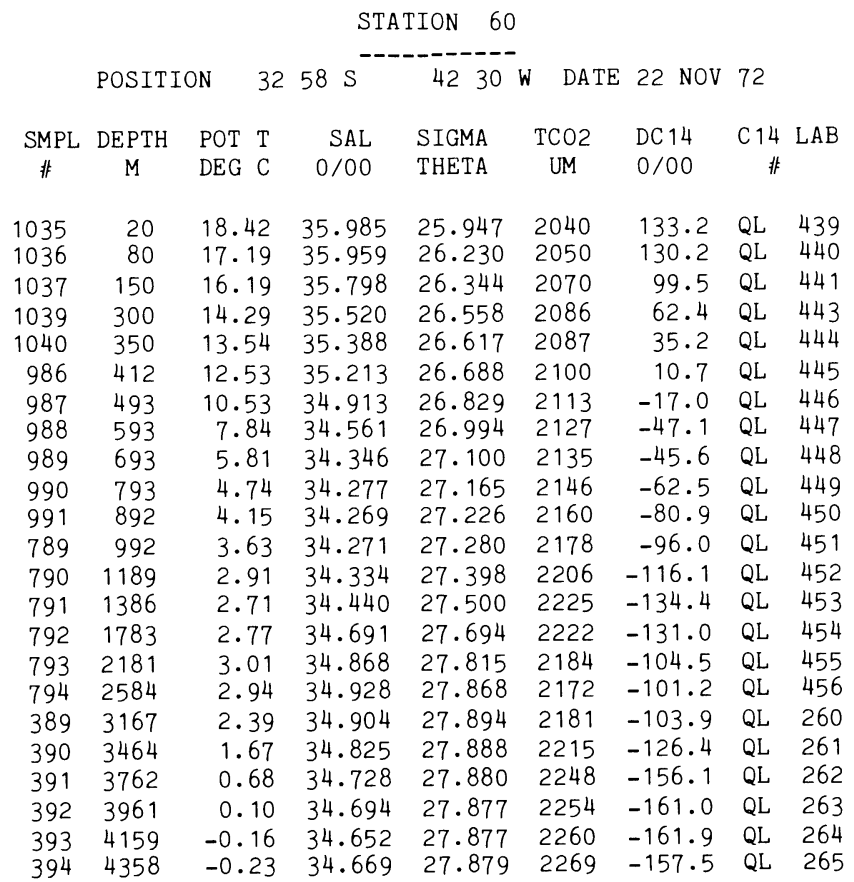




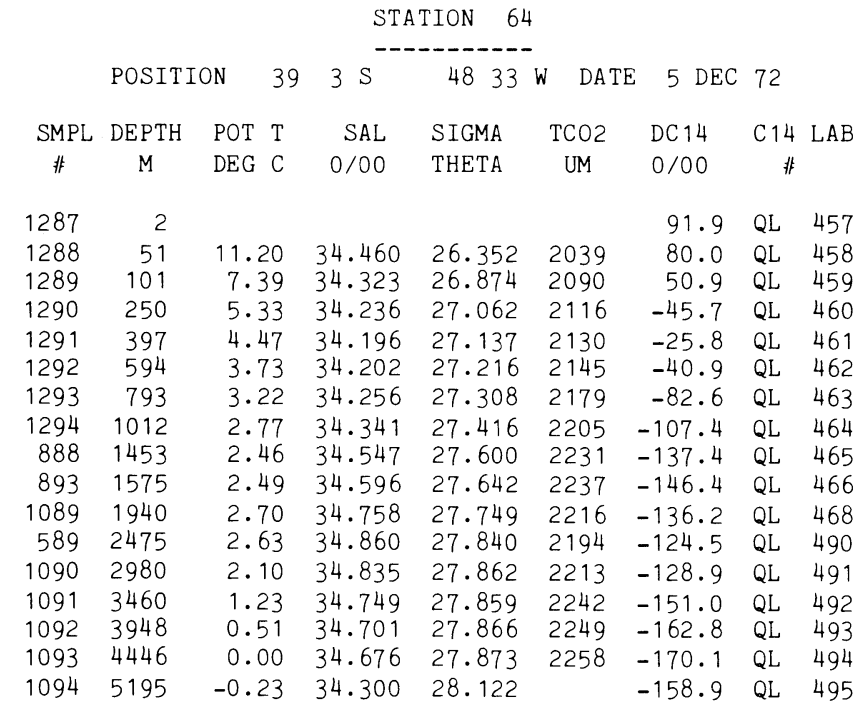

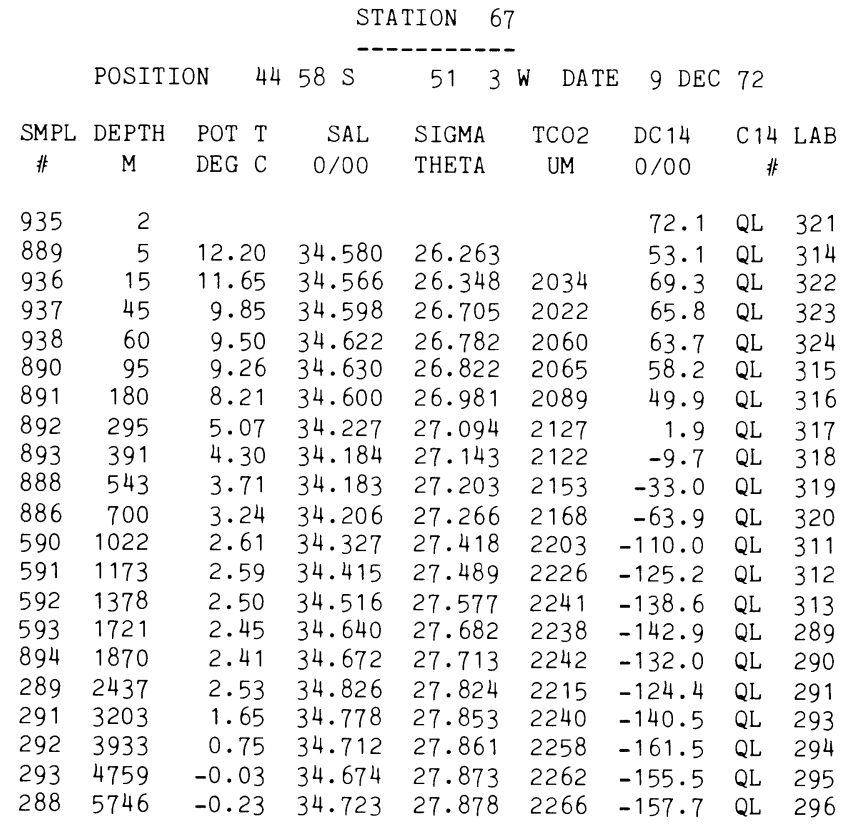




\begin{tabular}{|c|c|c|c|c|c|c|c|}
\hline \multicolumn{8}{|c|}{ STATION 68} \\
\hline POSIT & & $39 \mathrm{~s}$ & $45 \quad 59$ & DATE & E $13 \mathrm{DEC}$ & 72 & \\
\hline $\mathrm{EPTH}$ & POT & SAL & SIGMA & $\mathrm{TCO} 2$ & DC 14 & C 14 & $\mathrm{LAF}$ \\
\hline M & DEG & $0 / 00$ & THETA & UM & $0 / 00$ & 非 & \\
\hline 11 & 9.01 & 34.259 & 26.578 & & & ML & 940 \\
\hline 62 & $5.52-12$ & 34.199 & 27.0 & & 26 & ML & \\
\hline 340 & 3.21 & 34.1 & 27. & & -26 & $\mathrm{ML}$ & \\
\hline 530 & 2.77 & 34.264 & 27.35 & & -84.2 & ML & 936 \\
\hline 1450 & 2.49 & 34.700 & 27.726 & & -138.6 & ML & 94 \\
\hline 24 & 2.17 & 34. & 27.8 & 2232 & -136.5 & ML & 94 \\
\hline 2632 & 1.60 & 34.7 & 2 & 2244 & -147.0 & ML & 94 \\
\hline & -0.10 & & 27.875 & 2264 & -161.7 & ML & 94 \\
\hline 8 & -0.25 & 70 & 27.480 & & -155.4 & $\mathrm{ML}$ & \\
\hline
\end{tabular}

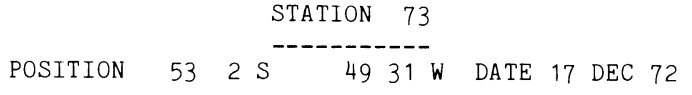

$\begin{array}{ccccccccc}\text { SMPL } & \text { DEPTH } & \text { POT } & \text { T } & \text { SAL } & \text { SIGMA } & \text { TC02 } & \text { DC14 } & \text { C14 LAB } \\ \text { \# } & \text { M } & \text { DEG } & \text { C } & 0 / 00 & \text { THETA } & \text { UM } & 0 / 00 & \text { \# }\end{array}$

$\begin{array}{rrrrrrrr}291 & 2 & & & & 4.7 & \text { QL } & 469 \\ 292 & 240 & 3.25 & 34.181 & 27.245 & 37.0 & \text { QL } & 470 \\ 293 & 488 & 2.60 & 34.228 & 27.339 & -75.2 & \text { QL } & 471\end{array}$




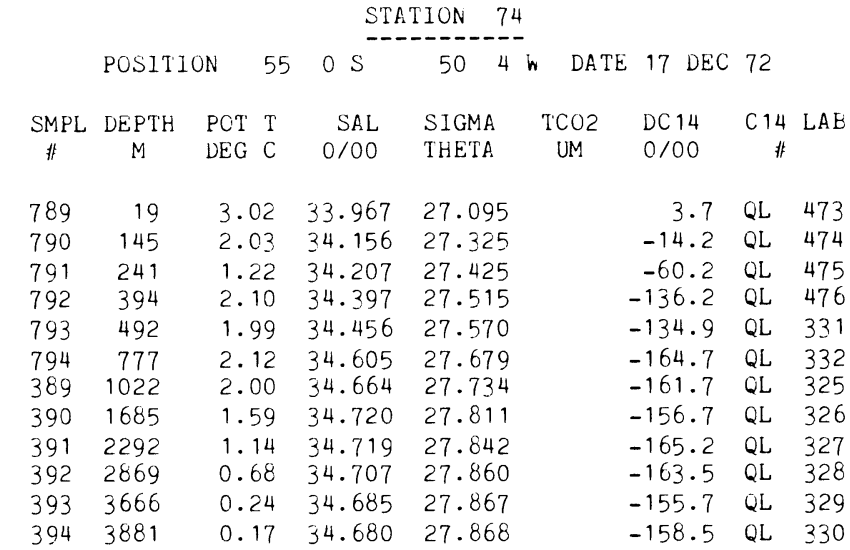

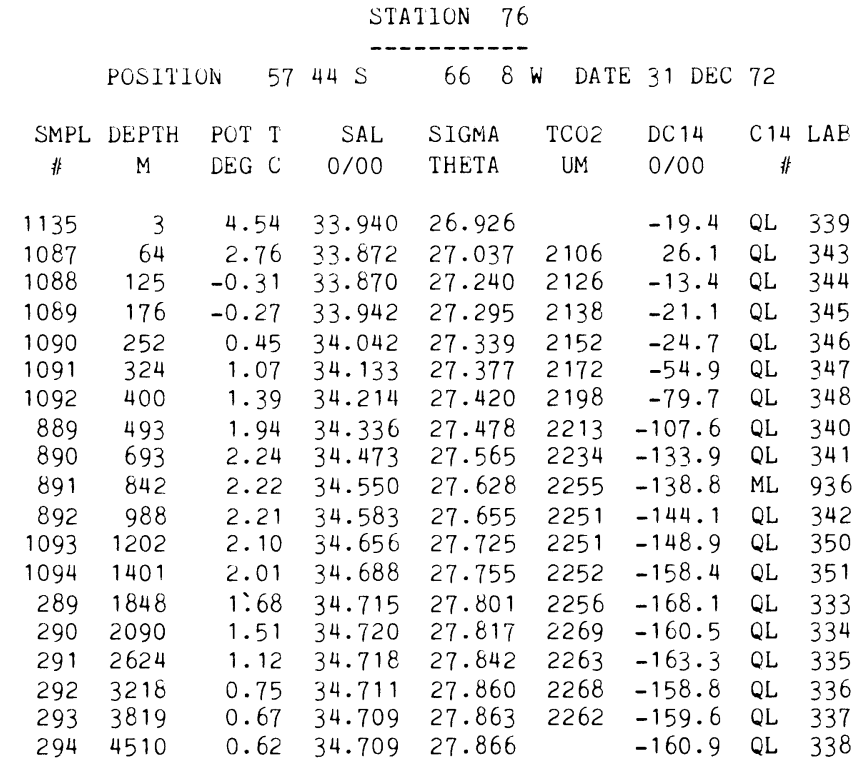

\begin{tabular}{|c|c|c|c|c|c|c|c|c|}
\hline \multirow{3}{*}{$\begin{array}{c}\text { SMPL } \\
\text { \#⿰ }\end{array}$} & \multicolumn{2}{|c|}{ POSITION } & $3 s$ & 6258 & DATE & $3 \mathrm{JAN}$ & 73 & \\
\hline & DEPTH & POT T & SAL & SIGMA & $\mathrm{TCO} 2$ & DC 14 & C 14 & $\mathrm{LAB}$ \\
\hline & M & DEG C & $0 / 00$ & THETA & UM & $0 / 00$ & 非 & \\
\hline 892 & 95 & -0.97 & 33.971 & $27 \cdot 346$ & 2151 & -18.6 & QL & 477 \\
\hline 893 & 142 & 0.50 & 34.153 & 27.425 & 2196 & $-62 \cdot 1$ & QL & 478 \\
\hline 894 & 189 & 1.33 & 34.279 & 27.476 & 2212 & -97.0 & $\mathrm{QL}$ & 479 \\
\hline 689 & 246 & 1.66 & 34.355 & 27.514 & 2223 & $-125 \cdot 3$ & QL & 480 \\
\hline 690 & 345 & 2.00 & 34.483 & 27.591 & 2247 & -132.2 & ML & 937 \\
\hline 691 & 445 & 2.10 & 34.554 & 27.641 & 2252 & -142.4 & $\mathrm{QL}$ & 481 \\
\hline 692 & 545 & 2.08 & 34.597 & 27.676 & 2256 & -145.8 & QL & 482 \\
\hline 693 & 671 & 2.04 & 34.644 & 27.717 & 2257 & -147.0 & QL & 483 \\
\hline 694 & 889 & 1.94 & 34.699 & 27.768 & 2254 & -155.4 & QL & 484 \\
\hline 389 & 1192 & 1.70 & 34.723 & 27.805 & 2253 & -161.9 & QL & 485 \\
\hline 390 & 1589 & 1.31 & 34.729 & 27.838 & 2258 & -160.6 & QL & 486 \\
\hline 391 & 1985 & 1.03 & 34.721 & 27.850 & 2265 & -163.2 & $\mathrm{QL}$ & 487 \\
\hline 393 & 2978 & 0.38 & 34.704 & 27.875 & 2262 & -161.9 & QL & 489 \\
\hline 394 & 3478 & 0.31 & 34.696 & 27.873 & 2265 & -163.3 & ML & 1056 \\
\hline
\end{tabular}




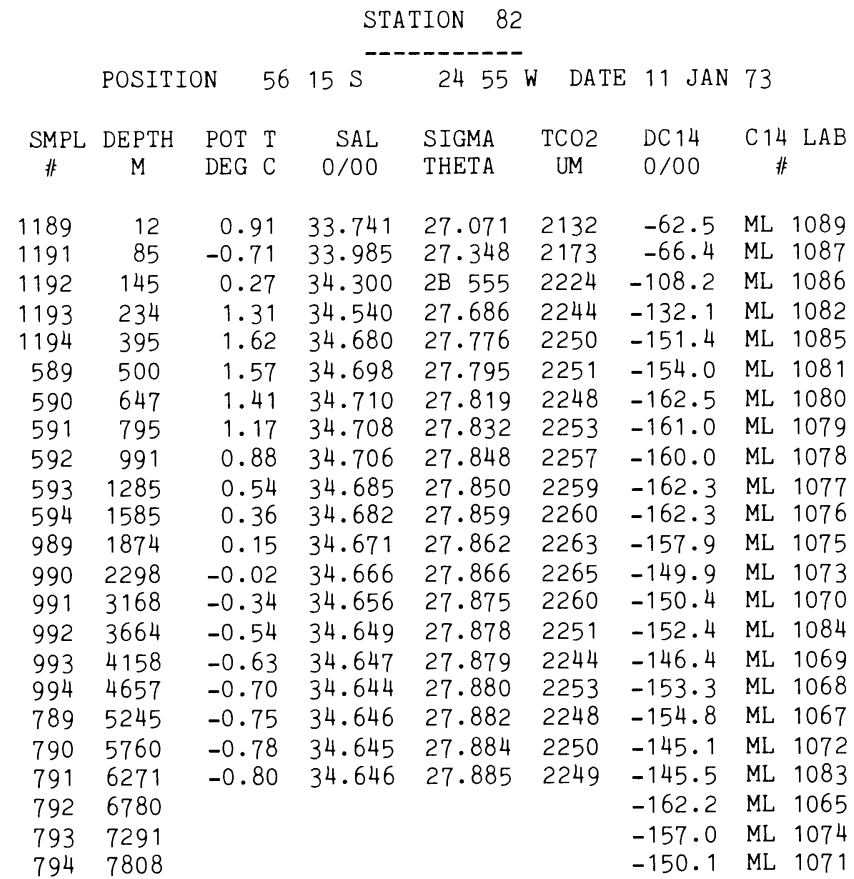

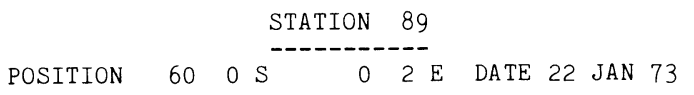

\begin{tabular}{|c|c|c|c|c|c|c|c|}
\hline SMPL & DEPTH & POT T & SAL & SIGMA & TCO2 & DC 14 & C14 LAB \\
\hline 非 & M & DEG C & $0 / 00$ & THETA & UM & $0 / 00$ & 非 \\
\hline 987 & 21 & 0.61 & 34.083 & 27.363 & 2158 & $-43 \cdot 5$ & ML \\
\hline 988 & 52 & -1.29 & 34.293 & 27.616 & 2199 & -59.7 & ML \\
\hline 989 & 105 & -1.66 & 34.417 & 27.729 & 2223 & -72.5 & ML \\
\hline 786 & 137 & -0.82 & 34.513 & 27.777 & 2237 & -74.7 & ML \\
\hline 787 & 199 & 0.45 & 34.662 & 27.841 & 2269 & -158.8 & ML 11 \\
\hline 788 & 400 & 0.44 & 34.685 & 27.857 & 2270 & $-164 \cdot 6$ & ML 110 \\
\hline 89 & 601 & 0.33 & 34.684 & 27.863 & 2275 & $-164 \cdot 5$ & ML 110 \\
\hline 790 & 797 & 0.23 & 34.678 & 27.863 & 2271 & -161.1 & ML 1 \\
\hline 791 & 1095 & 0.08 & 34.673 & 27.867 & 2267 & -156.4 & ML \\
\hline 866 & 1436 & -0.07 & 34.670 & 372 & 2262 & .4 & ML \\
\hline 587 & 1788 & -0.22 & 34.663 & 27.874 & 2249 & -166.8 & ML 109 \\
\hline 588 & 2134 & -0.34 & 34.661 & 27.877 & 2258 & -161.1 & ML 109 \\
\hline 89 & 2480 & -0.42 & 34.656 & 27.877 & 2250 & $-164 \cdot 5$ & ML \\
\hline 590 & 2832 & -0.50 & 34.654 & 27.879 & 2258 & -163.9 & ML \\
\hline 592 & 3184 & -0.56 & 34.652 & 27.880 & 2255 & -160.0 & ML \\
\hline 188 & 3883 & -0.71 & 34.651 & 27.885 & 2249 & -160.8 & ML \\
\hline 189 & 4230 & -0.80 & 34.650 & 27.888 & 2243 & -157.0 & ML \\
\hline & 4579 & -0.85 & 34.649 & 27.889 & 2246 & $-150 \cdot 1$ & ML \\
\hline & & -0.88 & 34.647 & 27.8 & 2245 & $-155 \cdot 5$ & ML \\
\hline 194 & 5268 & -0.89 & 34.633 & 28.037 & 2245 & -153.6 & ML 10 \\
\hline
\end{tabular}

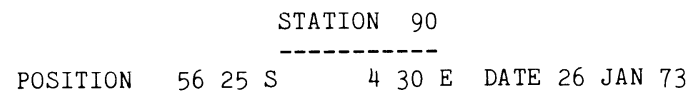

$\begin{array}{ccccccccc}\text { SMPL } & \text { DEPTH } & \text { POT } & \text { T } & \text { SAL } & \text { SIGMA } & \text { TCO2 } & \text { DC14 } & \text { C14 LAB } \\ \text { \# } & \text { M } & \text { DEG } & \text { C } & 0 / 00 & \text { THETA } & \text { UM } & 0 / 00 & \text { \#⿰ }\end{array}$

$\begin{array}{lllllllll}235 & 3 & 1.23 & 33.833 & 27.125 & 2129 & -55.7 & \text { ML } & 1983\end{array}$

$\begin{array}{lllllllll}236 & 50 & 1.05 & 33.856 & 27.166 & 2138 & -54.2 & \text { ML } 1982\end{array}$

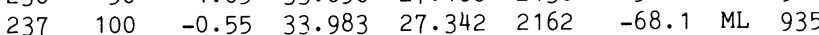

$\begin{array}{llllllll}238 & 130 & -0.37 & 34.133 & 27.453 & 2197 & -83.1 & \text { ML } 1981\end{array}$ 


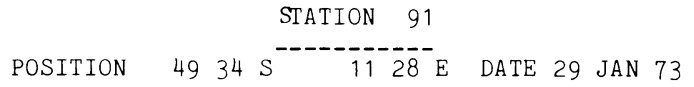

\begin{tabular}{|c|c|c|c|c|c|c|c|}
\hline $\begin{array}{l}\text { MPL } \\
\text { \# }\end{array}$ & $\underset{\mathrm{M}}{\text { DEPTH }}$ & $\begin{array}{ll}\text { POT } & \mathrm{T} \\
\text { DEG } & \mathrm{C}\end{array}$ & $\begin{array}{r}\text { SAL } \\
0 / 00\end{array}$ & $\begin{array}{l}\text { SIGMA } \\
\text { THETA }\end{array}$ & $\begin{array}{c}\mathrm{TCO} 2 \\
\mathrm{UM}\end{array}$ & $\begin{array}{l}\mathrm{DC} 14 \\
0 / 00\end{array}$ & $\begin{array}{c}\mathrm{C} 14 \mathrm{LAB} \\
\text { 非 }\end{array}$ \\
\hline 89 & 19 & 4.28 & 33.842 & 26.875 & 2103 & 18.6 & ML 11 \\
\hline 30 & 99 & & 5 & & & 10.6 & ML 112 \\
\hline 1 & 153 & .7 & & 27. & 2131 & 2.1 & ML 112 \\
\hline 2 & 346 & & & & 222 & 112.0 & ML 112 \\
\hline 93 & 545 & & 8 & & 224 & & ML 113 \\
\hline 94 & 746 & .12 & & & 2251 & & ML 1119 \\
\hline 39 & 988 & & & & & & ML 11 \\
\hline 90 & 1288 & & & & 2244 & -14 & ML 11 \\
\hline & & & & & 2237 & & ML 1117 \\
\hline 32 & 1783 & & & & & & ML 1116 \\
\hline 3 & & & & & 2252 & & ML 1118 \\
\hline 4 & & & & & & & ML 1115 \\
\hline 89 & 268 & & & & 225 & & ML 1113 \\
\hline & & & & & & & ML 1112 \\
\hline & 318 & & & & 22 & & ML 1135 \\
\hline & & & & & & & ML 111 \\
\hline & & & & & & & \\
\hline & 4085 & 0.20 & 34.679 & 27.865 & 2259 & -165.7 & IL \\
\hline
\end{tabular}

\begin{tabular}{|c|c|c|c|c|c|c|c|c|}
\hline \multirow{3}{*}{$\underset{\# \mathbb{P}}{S N_{i} P L}$} & POSI' & & 46 & $11 \mathrm{~s}$ & 1436 & DATE & $31 \mathrm{JAN}$ & 73 \\
\hline & DEPTH & POT & T' & SAL & SIGMA & $\mathrm{ICO} 2$ & DC 14 & C14 LAB \\
\hline & M & DEG & c & $0 / 00$ & THETA & UM & $0 / 00$ & $\sharp$ \\
\hline 235 & 3 & $7 \cdot 3$ & & 33.824 & 26.483 & 205 & 63.7 & ML 1987 \\
\hline 236 & 55 & 7.1 & & 33.824 & 26.511 & 2065 & 59.4 & ML 1986 \\
\hline 237 & 110 & 4.8 & & 33.909 & 26.868 & 2085 & 55.5 & ML 1985 \\
\hline 238 & 176 & 4.3 & & 34.115 & 27.089 & 2103 & 26.4 & ML 1984 \\
\hline
\end{tabular}

\begin{tabular}{|c|c|c|c|c|c|c|c|}
\hline & & & & ION & & & \\
\hline & POSIT] & ON & $46 s$ & 1827 & DATE & $2 \mathrm{FEB}$ & 73 \\
\hline SMPL & DEPTH & POT T & SAL & SIGMA & $\mathrm{TCO} 2$ & DC 14 & C14 LAB \\
\hline \# & M & DEG C & $0 / 00$ & THETA & UM & $0 / 00$ & \# \\
\hline 235 & 3 & 13.71 & 34.851 & 26.167 & & 89.8 & ML 1992 \\
\hline & 86 & 13.17 & 35.067 & 26.450 & 2045 & 77.2 & ML 1148 \\
\hline 587 & 244 & 10.26 & 34.739 & 26.743 & 2087 & 36.1 & ML 1147 \\
\hline 588 & 394 & 8.88 & 34.626 & 26.885 & 2108 & 10.2 & ML 1146 \\
\hline 589 & 740 & 4.58 & 34.331 & 27.231 & 2167 & -86.2 & ML 1145 \\
\hline $\begin{array}{l}590 \\
591\end{array}$ & $\begin{array}{l}1036 \\
1283\end{array}$ & $\begin{array}{l}3.51 \\
2.99\end{array}$ & $\begin{array}{l}34.437 \\
34.553\end{array}$ & $\begin{array}{l}27.424 \\
27.565\end{array}$ & $\begin{array}{l}2224 \\
2247\end{array}$ & $\begin{array}{l}-114.0 \\
-138.4\end{array}$ & $\begin{array}{ll}\text { ML } & 1144 \\
\text { ML } & 1143\end{array}$ \\
\hline 592 & 1579 & 2.67 & 34.671 & 27.687 & 2245 & -142.8 & ML 1141 \\
\hline 593 & 1878 & 2.55 & 34.763 & 27.772 & 2230 & -130.8 & ML 1142 \\
\hline 594 & 2277 & 2.39 & 34.809 & 27.822 & 2219 & -131.2 & ML 1132 \\
\hline 386 & 2681 & 2.16 & 34.823 & 27.850 & 2224 & -120.8 & ML 1140 \\
\hline 387 & 2884 & 2.07 & & 27.859 & 2209 & -129.0 & ML 1139 \\
\hline 388 & 3082 & 1.90 & & 27.863 & 2225 & -132.5 & ML 1131 \\
\hline 389 & 3281 & 1.81 & 34.808 & 27.869 & 2229 & -134.5 & ML 1130 \\
\hline 390 & 3484 & 1.60 & 34.796 & 27.872 & 2230 & -132.4 & ML 1128 \\
\hline & 3684 & 1.32 & 34.769 & 27.869 & 2242 & -141.9 & ML 1129 \\
\hline 392 & 3980 & 0.95 & 34.738 & 27.869 & 2259 & -149.5 & ML 1127 \\
\hline 393 & 4279 & 0.68 & 34.719 & 27.870 & 2255 & -151.6 & ML 1126 \\
\hline 39 & 4625 & & & & & -160.9 & ML 1138 \\
\hline
\end{tabular}




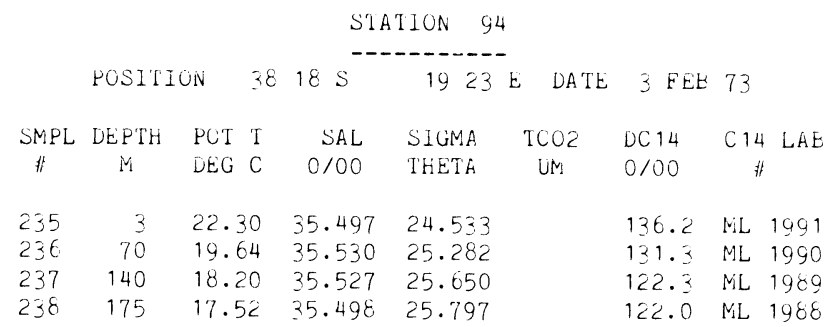

POSITION $2359 \mathrm{~S} \quad 830$ E DATE 17 FEB 73

\begin{tabular}{|c|c|c|c|c|c|c|c|c|}
\hline SMPL & DEPTH & POT T & SAL & SIGMA & TCO2 & DC 14 & C 14 & LAB \\
\hline 非 & $\mathrm{M}$ & DEG $C$ & $0 / 00$ & THETA & UM & $0 / 00$ & 非 & \\
\hline 535 & 3 & 23.42 & 35.591 & 24.282 & & 74.4 & QL & 496 \\
\hline 786 & 93 & 16.02 & 35.492 & 26.155 & 2057 & 101.0 & QL & 497 \\
\hline 787 & 142 & 15.03 & 35.411 & 26.311 & 2071 & 90.6 & QL & 498 \\
\hline 788 & 192 & 13.17 & 35.151 & 26.510 & 2094 & 57.4 & QL & 499 \\
\hline 789 & 291 & 11.09 & 34.953 & 26.761 & 2151 & -6.7 & QL & 500 \\
\hline 790 & 391 & 8.79 & 34.717 & 26.972 & 2198 & -49.2 & QL & 501 \\
\hline 791 & 489 & 7.22 & 34.589 & 27.105 & 2224 & -81.2 & QL & 502 \\
\hline 792 & 690 & 5.03 & 34.461 & 27.285 & 2231 & $-108 \cdot 5$ & QL & 503 \\
\hline 793 & 888 & 3.96 & 34.472 & 27.409 & 2231 & -120.0 & QL & 504 \\
\hline 794 & 1088 & 3.49 & 34.561 & 27.525 & 2232 & $-125 \cdot 9$ & QL & 505 \\
\hline 286 & 1368 & 3.27 & 34.724 & 27.676 & 2228 & -126.5 & QL & 506 \\
\hline 288 & 1964 & 3.02 & 34.889 & 27.830 & 2194 & -109.0 & QL & 507 \\
\hline 290 & 2958 & 2.24 & 34.862 & 27.875 & 2209 & $-125 \cdot 5$ & QL & 508 \\
\hline 291 & 3454 & 2.03 & 34.849 & 27.883 & 2224 & -125.6 & ML & 1152 \\
\hline 292 & 3949 & 1.43 & 34.796 & 27.884 & 2239 & -144.1 & ML & 1150 \\
\hline 293 & 4248 & 0.85 & 34.743 & 27.879 & 2256 & -156.9 & QL & 509 \\
\hline 294 & 4546 & 0.72 & 34.836 & 27.881 & 2263 & -155.4 & ML & 1149 \\
\hline
\end{tabular}

\begin{tabular}{|c|c|c|c|c|c|c|c|c|}
\hline & & & & ION 10 & & & & \\
\hline & POSIT & & $0 \mathrm{~S}$ & 2 & DATE & 22 FEB & 73 & \\
\hline SMPL & DEPTH & POT 1 & SAL & SIGMA & TCO2 & DC 14 & C 14 & LAB \\
\hline 非 & M & DEG $C$ & $0 / 00$ & THETA & UM & $0 / 00$ & 非 & \\
\hline 435 & 2 & & & & & 56.1 & QL & 510 \\
\hline 686 & 48 & 22.48 & 36.580 & 25.298 & 2065 & 64.4 & QL & 511 \\
\hline 687 & 98 & 15.78 & 35.693 & 26.343 & 2160 & -10.2 & $\mathrm{QL}$ & 512 \\
\hline 688 & 147 & 12.49 & 35.225 & 26.705 & 2214 & -56.2 & $\mathrm{QL}$ & 513 \\
\hline 689 & 217 & 11.04 & 35.045 & 26.842 & 2231 & -69.0 & QL & 514 \\
\hline 690 & 346 & 9.42 & 34.857 & 26.978 & 2242 & -82.9 & QL & 515 \\
\hline 691 & 594 & 6.04 & 34.559 & 27.241 & 2262 & -105.9 & QL & 516 \\
\hline 692 & 893 & 4.27 & 34.520 & 27.415 & 2247 & $-120 \cdot 7$ & QL & 517 \\
\hline 693 & 1092 & 3.92 & 34.633 & 27.540 & 2237 & $-124 \cdot 6$ & QL & 518 \\
\hline 694 & 1390 & 3.68 & 34.830 & 27.711 & 2218 & -110.8 & QL & 519 \\
\hline 286 & 1775 & 3.40 & 34.909 & 27.805 & 2194 & -101.1 & QL & 520 \\
\hline 287 & 2073 & 3.02 & 34.921 & 27.851 & 2196 & -107.4 & QL & 521 \\
\hline 288 & 2571 & 2.65 & 34.911 & 27.880 & 2205 & -118.0 & QL & 522 \\
\hline 289 & 3069 & 2.30 & 34.896 & 27.897 & 2212 & -124.6 & QL & 523 \\
\hline 290 & 3565 & 2.10 & 34.889 & 27.908 & 2222 & -125.4 & $\mathrm{QL}$ & 524 \\
\hline 291 & 4061 & 2.01 & 34.885 & 27.912 & 2222 & -119.4 & QL & 525 \\
\hline 292 & 4557 & 1.97 & 34.879 & 27.910 & 2216 & $-123 \cdot 9$ & QL & 526 \\
\hline 293 & 5055 & 1.94 & 34.878 & 27.912 & 2224 & -118.2 & QL & 527 \\
\hline 294 & 5551 & 1.79 & 34.877 & 27.309 & 2215 & -117.9 & QL & 528 \\
\hline
\end{tabular}




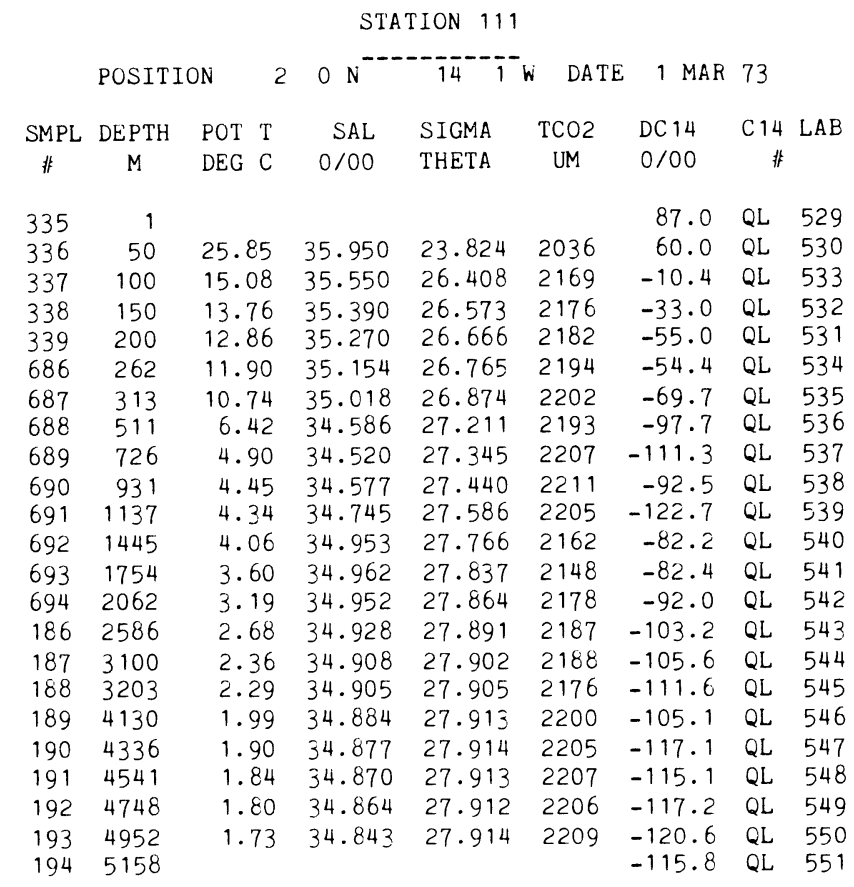

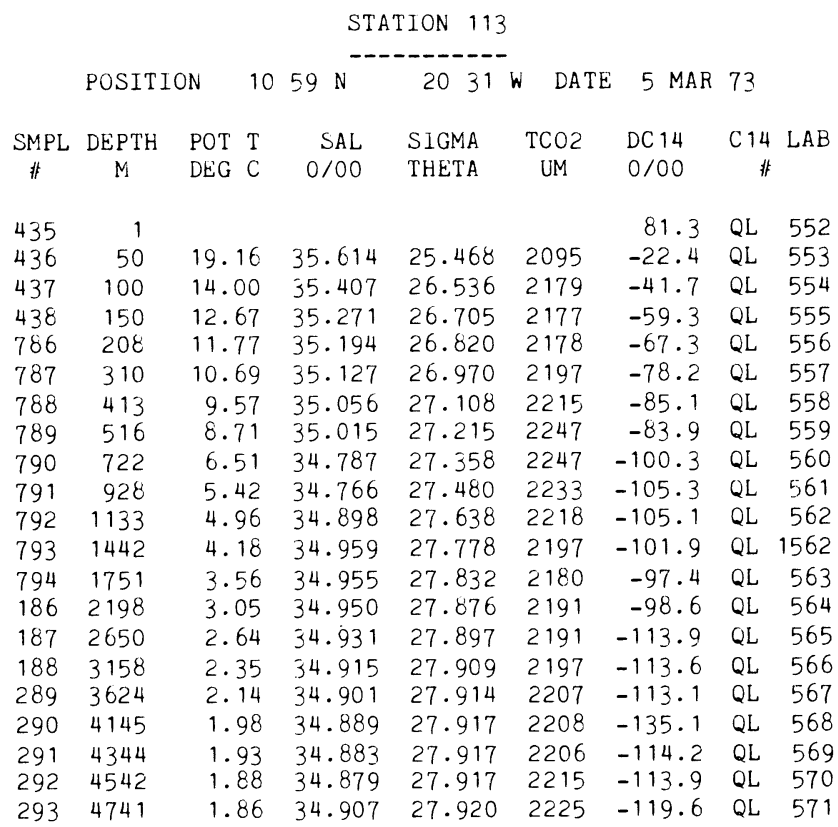




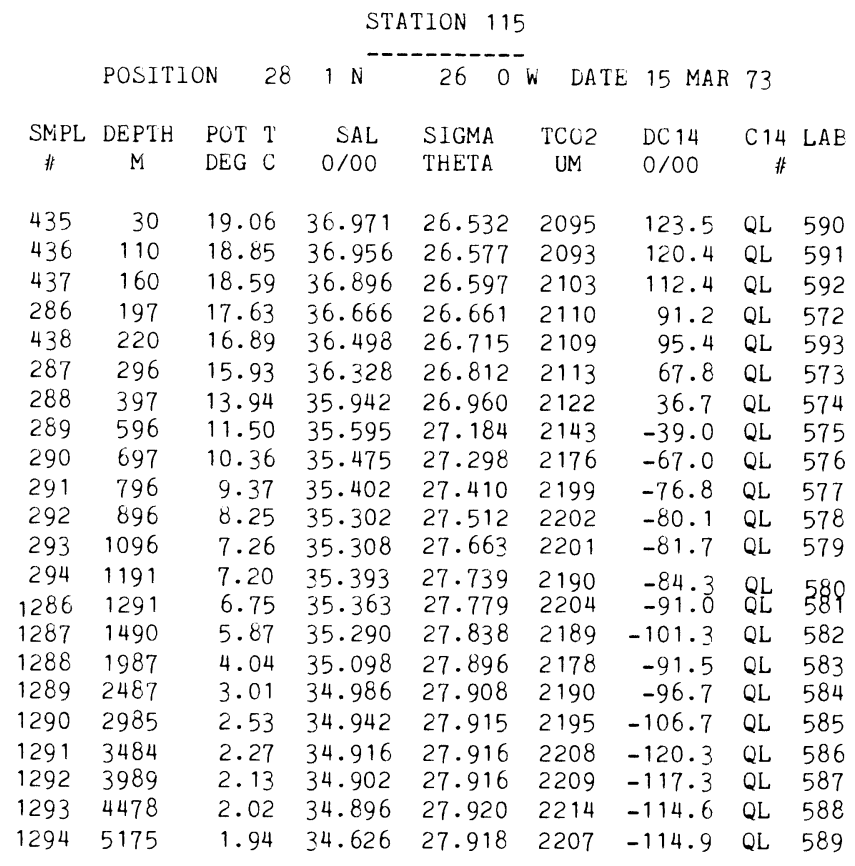

\begin{tabular}{|c|c|c|c|c|c|c|c|c|c|}
\hline \multirow[b]{3}{*}{ SMPI } & \multicolumn{9}{|c|}{ STATION 117} \\
\hline & \multicolumn{3}{|c|}{ POSITION } & $40 \mathrm{~N}$ & 3858 & DATE & 20 MAR & 73 & \\
\hline & DEPTH & POT & $\mathrm{T}$ & SAL & SIGMA & $\mathrm{TCO} 2$ & DC 14 & C 14 & $\mathrm{LAB}$ \\
\hline \# & M & DEG & C & $0 / 00$ & THETA & UM & $0 / 00$ & \# & \\
\hline 786 & 2 & & & & & & 138.9 & QL & 594 \\
\hline 787 & 100 & 19.19 & & 36.673 & 26.276 & 2065 & 136.0 & QL & 595 \\
\hline 788 & 295 & 17.00 & & 36.403 & 26.615 & 2096 & 79.8 & QL & 596 \\
\hline 789 & 393 & 16.08 & & 36.230 & 26.702 & 2102 & 48.9 & QL & 597 \\
\hline 790 & 539 & 13.8 & & 35.871 & 26.933 & 2120 & 11.2 & $Q L$ & 598 \\
\hline 791 & 686 & 11.84 & & 35.609 & 27.129 & 2148 & -32.2 & QL & 599 \\
\hline 793 & 1076 & 7.28 & & 35.285 & 27.644 & 2188 & -67.9 & QL & 600 \\
\hline 286 & 1215 & 6.43 & & 35.251 & 27.734 & 2183 & -71.2 & QL & 601 \\
\hline 287 & 1319 & 5.81 & & 35.224 & 27.793 & 2176 & -75.3 & QL & 602 \\
\hline 288 & 1474 & 5.14 & & 35.175 & 27.836 & 2171 & $-72 \cdot 3$ & QL & 603 \\
\hline 289 & 1681 & 4.50 & & 35.134 & 27.877 & 2174 & -78.0 & $\mathrm{QL}$ & 604 \\
\hline 794 & 1954 & 3.78 & & 35.059 & 27.893 & 2170 & -78.3 & QL & 605 \\
\hline 291 & 2506 & 2.90 & & 34.964 & 27.901 & 2172 & -92.1 & QL & 606 \\
\hline 292 & 3019 & 2.58 & & 34.941 & 27.910 & 2193 & -104.0 & QL & 607 \\
\hline 293 & 3425 & 2.43 & & 34.929 & 27.914 & 2194 & -105.0 & QL & 608 \\
\hline
\end{tabular}




POSITION $3316 \mathrm{~N}$ STATION 120

\begin{tabular}{|c|c|c|c|c|c|c|c|c|}
\hline SMPL & DEPTH & POT $\mathrm{T}$ & SAL & SIGMA & TCO2 & DC 14 & C 14 & LAB \\
\hline \# & M & DEG C & $0 / 00$ & THETA & UM & $0 / 00$ & 非 & \\
\hline 286 & 3 & 18.04 & 36.417 & 26.370 & 2052 & 127.8 & QL & 609 \\
\hline 287 & 204 & 17.65 & 36.388 & 26.444 & 2067 & 129.5 & QL & 610 \\
\hline 288 & 403 & 15.94 & 36.167 & 26.687 & 2101 & 45.6 & QL & 611 \\
\hline 289 & 601 & 12.67 & 35.652 & 26.998 & 2131 & -12.1 & QL & 612 \\
\hline 290 & 700 & 10.56 & 35.366 & $27 \cdot 178$ & 2162 & -59.7 & QL & 13 \\
\hline 291 & 799 & 8.99 & 35.218 & $27 \cdot 331$ & 2174 & -66.0 & QL & 14 \\
\hline 292 & 899 & 7.17 & 35.095 & 27.510 & 2194 & -62.0 & QL & 15 \\
\hline 293 & 1096 & 5.57 & 35.085 & 27.715 & 2187 & -61.4 & QL & 616 \\
\hline 294 & 1293 & 4.91 & 35.066 & 27.778 & 2170 & -62.4 & QL & 617 \\
\hline 586 & 1757 & 3.94 & 35.011 & 27.836 & 2155 & -68.0 & QL & 618 \\
\hline 587 & 2255 & 3.40 & 34.983 & 27.869 & 2169 & -75.8 & QL & 619 \\
\hline 588 & 2754 & 2.87 & 34.954 & 27.895 & 2169 & -87.8 & QL & 620 \\
\hline 589 & 3252 & 2.46 & 34.928 & 27.910 & 2168 & -91.6 & QL & 621 \\
\hline 590 & 3550 & 2.21 & 34.915 & 27.920 & 2171 & $-88 \cdot 3$ & QL & 622 \\
\hline 591 & 4048 & 1.95 & 34.895 & $27 \cdot 925$ & 2182 & -93.4 & QL & 623 \\
\hline 592 & 4793 & 1.75 & 34.874 & $27 \cdot 923$ & 2185 & $-100 \cdot 1$ & QL & 624 \\
\hline 594 & 5535 & 1.65 & 36.596 & 27.957 & 2201 & -101.4 & $Q L$ & 625 \\
\hline
\end{tabular}

